\title{
Symmetry-breaking waves and space-time modulation mechanisms in two-dimensional plane Poiseuille flow
}

\author{
Roger Ayats and Alvaro Meseguer* \\ Department of Physics, Universitat Politècnica de Catalunya, 08034 Barcelona, Spain \\ Fernando Mellibovsky \\ Department of Physics - Aerospace Engineering Division, \\ Universitat Politècnica de Catalunya, 08034, Barcelona \\ (Dated: September 2, 2020)
}




\begin{abstract}
We investigate two distinct scenarios of spatial modulation that are candidate mechanisms for streamwise localisation of waves in two-dimensional plane Poiseuille flow. The first one stems from a symmetry-breaking bifurcation that disrupts the half-shift \& reflect equivariance of TollmienSchlichting waves (TSW). A new state, an asymmetric TSW (ATSW), emerges from unstable lowerbranch Tsws at subcritical Reynolds number and undergoes subharmonic Hopf bifurcations that lead to branches of asymmetric time-periodic space-modulated waves (MATSW). Streamwise modulation does not evolve into localisation within the range of parameters explored. In breaking the last standing remnants of the reflectional symmetry about the channel midplane, ATSW and MATSW admit a bias towards either one of the channel walls, thus bearing a potential for explaining near-wall structures that are typical of developed turbulence. The second scenario follows the fate of a branch of time-periodic space-modulated TSWs (MTSW) initially discovered by Mellibovsky \& Meseguer [J. Fluid Mech. 779, R1 (2015)]. We find that these waves can lead to localisation but the mechanism is not new, as they do so through their connection, by means of a codimension-2 bifurcation point, with other known localising MTSws. The codimension-2 point is however responsible for the appearance of MTSWs that exclusively bridge upper-branch TSW-trains of different number of replicas. In this respect, these MTSWs possess all required properties that single them out as possible constituents of the strange saddle that governs domain-filling turbulent dynamics at high Reynolds numbers.
\end{abstract}

\footnotetext{
${ }^{*}$ Corresponding author: alvaro.meseguer@upc.edu
} 


\section{INTRODUCTION}

Subcritical transition and intermittency phenomena in wall-bounded shear flows remains one of the most intriguing and still unsolved conundrums in fluid dynamics $[2,3]$. Understanding and predicting when the flow in pipes and channels becomes unstable and eventually turbulent is of the utmost importance to many branches of science and technology, yet shear flow transition often occurs at moderate Reynolds numbers for which the underlying laminar base flow is linearly stable $[4,5]$. Nonlinear instabilities and transition in shear flows have been intensively studied over the last couple of decades, mainly following the identification of exact solutions to the Navier-Stokes equations in pipe flow [6-9], plane Couette flow [10-14] or plane Poiseuille flow $[1,15]$, this latter henceforth referred to as PPF. These solutions are exact coherent flow states, travelling waves in the case of PPF, that start appearing out of the blue at finite Reynolds number in saddle-node bifurcations. When increasing the Reynolds number, the nodal or upper branch typically exhibits secondary bifurcations that lead to increasingly complex flows featuring time-periodicity, quasi-periodicity and eventually chaotic and turbulent dynamics. Although bifurcated nodal solutions resulting from local bifurcations must in principle remain locally attracting, experiments and numerical simulations reveal that turbulent dynamics is transient $[16,17]$ at low to moderate Reynolds numbers. The eventual relaminarization of turbulent flow is a consequence of a global bifurcation involving a boundary crisis [18], whereby the phase-space boundary of the chaotic attractor resulting from successive bifurcations of the nodal branch collides with the saddle or some solution branch bifurcated from it, thus puncturing the local attractor and morphing it into a strange saddle. This motivates a careful inspection of the bifurcations undergone by the unstable saddle or lower branch of travelling wave solutions, which is a challenging computational task. The main reason is that these travelling waves are highly unstable so they cannot be identified by simply time-stepping the Navier-Stokes equations. The analysis requires instead the use of continuation algorithms purposely designed to accurately compute and track the unstable travelling waves in parameter space. Relevant portions of their eigenspectrum can then be computed via linear stability analysis with matrix-free Arnoldi-iteration and monitored to detect secondary bifurcations.

One of the most common and intriguing features of shear-flow turbulence is its localised nature. For low or even moderate Reynolds numbers, turbulence typically appears in lo- 
calised patches of a finite extent surrounded by laminar flow. Experiments and direct numerical simulations in pipe, channel and annular flows have systematically reported localised turbulence in the form of puffs or turbulent spots [19-23]. The underlying mechanisms responsible for localisation cannot be merely explained in terms of spatially periodic coherent states such as the aforementioned travelling waves. Pipe flow simulations [24] have identified exact Navier-Stokes solutions in the form of localised time-periodic travelling waves that govern the dynamics on the basin boundary that separates laminar steady flows from localised turbulence. In this sense, they constitute an edge-state, whose stable manifold separates, if only locally, laminar and turbulent dynamics. These solutions appear in saddle-node bifurcations, such that the nodal solution is an essential constituent of localised turbulent dynamics. The mechanism underlying pipe flow localisation was first described by [25] in terms of subharmonic instabilities connecting travelling-wave solution branches of different wavelengths. Along the same lines, [1], MM15 for short, studied the localisation mechanism in two-dimensional PPF, where stable/upper-branch Tsws become unstable to perturbations of wavelength longer than their own. Both time and space modulation arises from these subharmonic Hopf bifurcations, such that the resulting states include propagating wave dislocations. Continuation to larger fundamental wavelength of these new solutions often results in the eventual accommodation of an extra travelling wave replica that fills the gap generated by the dislocation and the branch lands anew on the family of Tsws at another subharmonic Hopf bifurcation of the lower/unstable branch. Occasionally, for low Reynolds numbers, the dislocation leaves a gap that widens indefinitely and the states become fully localised no matter how large the domain size grows. In this case, asymptotic analysis has recently shown that the upstream and downstream interfaces of the resulting localised patch decay exponentially towards the base parabolic flow [26].

MM15 carried the issue of localisation further to test what was left of the so-called snakes \& ladders scenario observed in plane Couette spanwise localisation [12] among many other extended problems featuring a $Z_{2}$ symmetry, in a system, PPF, where this symmetry is completely broken. They concluded that a mechanism similar to streamwise snaking was still possible, although structurally unstable with respect to small variations in domain periodicity length.

We focus here on the systematic exploration of two phenomena conspicuously overlooked by MM15. On the one hand, we search for travelling and time-periodic-travelling solution 
branches breaking the half-shift \& reflect symmetry of all known Tollmien-Schlichting waves (TSW) and the related half-period \& reflect equivariance of bifurcating time-periodic spacemodulated TSWs (MTSW). On the other hand, we investigate the fate of a family of MTSWs that allegedly had no bearing in the localisation mechanism investigated there. To do so, we perform a comprehensive linear stability analysis of the lower branch of Tsws with respect to disturbances of their same wavelength (superharmonic), but also, and most importantly, with respect to disturbances of wavelengths that are integer multiples of their own (subharmonic) in streamwise-replicated computational domains. This is systematically done for TSWs of all possible wavelengths and for a wide range of Reynolds numbers, with the aim of identifying and monitoring all subharmonic and symmetry-breaking bifurcations potentially leading to hitherto unknown branches of travelling or time-periodic-travelling waves.

The paper is structured as follows. In section $\S I I$ we provide an accurate description of the mathematical formulation of the problem. Section $\S I I I$ starts with a quick review of all known TSWs, along with an analysis of their stability and bifurcation, both subharmonic and superharmonic. Section $\S I V$ then dives into the search, parametric continuation, and subharmonic stability analysis of asymmetric travelling wave solutions (ATSW) emerging from the lower branch of TSWs, and provides a full description of the pitchfork bifurcations whereby their half-shift \& reflect symmetry is broken. Section $\S \mathrm{V}$ is devoted to a systematic exploration of the time-periodic space-modulated asymmetric waves (MATSW) that emerge from subharmonic Hopf bifurcations of ATSWs. In section §VI we track modulated-wave solution branches that had not been previously investigated and thoroughly unfold the codimension-2 bifurcation through which they are related to all other known branches of modulated waves. Finally, we outline the main results and summarize our conclusions in section $\S \mathrm{VII}$.

\section{MATHEMATICAL FORMULATION}

We consider an incompressible Newtonian fluid of kinematic viscosity $\nu$ and density $\rho$ confined in between two infinite parallel plates $2 h$ apart ( $h$ is the half-gap). The two-dimensional domain in cartesian coordinates is therefore $(x, y) \in(-\infty, \infty) \times[-h, h]$. Following Mm15,

the flow is driven at constant mass flux in order to better mimic the conditions found in experimental setups for extended systems such as pipes or channels [27, 28]. 
The flow is governed by the Navier-Stokes equations

$$
\partial_{t} \mathbf{u}+(\mathbf{u} \cdot \nabla) \mathbf{u}=-\frac{1}{\rho} \nabla p+\nu \nabla^{2} \mathbf{u}-\frac{\Pi}{\rho} \hat{\mathbf{x}}, \quad \nabla \cdot \mathbf{u}=0,
$$

where $\mathbf{u}=(u, v)$ and $p$ are the velocity and pressure fields, respectively. In (1), $\Pi$ is the streamwise driving pressure gradient, that is instantly adjusted to satisfy the prescribed constant mass flux. No-slip boundary conditions are imposed at the walls, $\mathbf{u}(x, \pm h ; t)=$ $(0,0)$, while the flow is assumed periodic in the streamwise direction $[\mathbf{u}, p](x+2 \pi / k, y ; t)=$ $[\mathbf{u}, p](x, y ; t)$, where $k$ is the streamwise wavenumber.

All variables are rendered dimensionless using $h$ and $3 U / 2$ as length and velocity scales, respectively, where $U$ is the mean streamwise velocity. Accordingly, the Reynolds number is defined as $\operatorname{Re}=3 h U / 2 \nu$ and the basic laminar solution of PPF is the streamwise-independent parabolic velocity profile $\mathbf{u}_{0}=\left(1-y^{2}\right) \hat{\mathbf{x}}$. The constant mass flux constraint is straightforwardly implemented by formulating the problem in the streamfunction formalism, so that (1) reads

$$
\partial_{t} \nabla^{2} \Psi+\left(\partial_{y} \Psi\right) \partial_{x}\left(\nabla^{2} \Psi\right)-\left(\partial_{x} \Psi\right) \partial_{y}\left(\nabla^{2} \Psi\right)=\frac{1}{\operatorname{Re}} \nabla^{4} \Psi
$$

where $\Psi(x, y ; t)$ is the non-dimensional streamfunction. Equation (2) is supplemented with no-slip boundary conditions at the walls, along with periodic boundary conditions in the streamwise coordinate

$$
\partial_{y} \Psi(x, \pm 1 ; t)=\partial_{x} \Psi(x, \pm 1 ; t)=0, \quad \Psi\left(x+\frac{2 \pi}{k}, y ; t\right)=\Psi(x, y ; t) .
$$

By arbitrarily choosing $\Psi(x, 1 ; t)=2 / 3$ and $\Psi(x,-1 ; t)=-2 / 3$, the volumetric flow rate condition

$$
\mathrm{Q}=\Psi(x, 1 ; t)-\Psi(x,-1 ; t)=4 / 3
$$

ensures the constant non-dimensional mean axial velocity is exactly and permanently $2 / 3$. In this formulation, the basic laminar PPF solution is expressed as $\Psi_{0}(y)=y\left(1-y^{2} / 3\right)$.

We analyse the effect of finite amplitude perturbations $\widetilde{\Psi}(x, y ; t)$ added to the basic PPF

$$
\Psi(x, y ; t)=\Psi_{0}(y)+\widetilde{\Psi}(x, y ; t)
$$

where $\widetilde{\Psi}$ satisfies periodic boundary conditions in the streamwise coordinate, along with homogeneous Neumann-Dirichlet boundary conditions in the wall-normal direction:

$$
\widetilde{\Psi}\left(x+\frac{2 \pi}{k}, y ; t\right)=\widetilde{\Psi}(x, y ; t), \quad \widetilde{\Psi}(x, \pm 1 ; t)=\partial_{x} \widetilde{\Psi}(x, \pm 1 ; t)=\partial_{y} \widetilde{\Psi}(x, \pm 1 ; t)=0 .
$$


Formal substitution of the perturbed field (5) in (2) yields

$$
\partial_{t} \nabla^{2} \widetilde{\Psi}=\mathbf{L}_{\operatorname{Re}}(\widetilde{\Psi})+\mathbf{N}(\widetilde{\Psi})
$$

where the linear biharmonic-advective and nonlinear convective terms are

$$
\left\{\begin{aligned}
\mathbf{L}_{\operatorname{Re}}(\widetilde{\Psi}) & =\frac{1}{\operatorname{Re}} \nabla^{4} \widetilde{\Psi}-\left(1-y^{2}\right) \partial_{x} \nabla^{2} \widetilde{\Psi}-2 \partial_{x} \widetilde{\Psi} \\
\mathbf{N}(\widetilde{\Psi}) & =\left(\partial_{x} \widetilde{\Psi}\right) \partial_{y} \nabla^{2} \widetilde{\Psi}-\left(\partial_{y} \widetilde{\Psi}\right) \partial_{x} \nabla^{2} \widetilde{\Psi}
\end{aligned}\right.
$$

The streamfunction appearing in (7) is spatially discretized within the computational domain $\Omega=\{(x, y) \in[0, \Lambda] \times[-1,1]\}$ using a truncated Fourier-Legendre spectral approximation in the $x-y$ coordinates, respectively, of the form

$$
\widetilde{\Psi}_{L M}(x, y ; t)=\sum_{\ell=-L}^{L} \sum_{m=0}^{M} a_{\ell m}(t) \Psi_{\ell m}(x, y),
$$

where

$$
\Psi_{\ell m}(x, y)=\mathrm{e}^{\mathrm{i} \ell k x} \Phi_{m}(y)
$$

and

$$
\Phi_{m}(y)=\left(1-y^{2}\right)^{2} \mathrm{~L}_{m}(y)
$$

with $\mathrm{L}_{m}(y)$ the $m^{\text {th }}$ Legendre polynomial. In the Fourier-Legendre spectral expansion above, $k=2 \pi / \Lambda$ is the fundamental streamwise wavenumber. As a consequence $\widetilde{\Psi}_{L M}$ is $\Lambda$-periodic in the $x$-streamwise coordinate, that is,

$$
\widetilde{\Psi}_{L M}(x+\Lambda, y ; t)=\widetilde{\Psi}_{L M}(x, y ; t) \quad \forall x
$$

Since $\widetilde{\Psi}_{L M} \in \mathbb{R}$, the set of Fourier-Legendre coefficients $\mathbf{a}=\left\{a_{\ell m}\right\}$ satisfy $a_{-\ell m}=a_{\ell m}^{*}$, where * denotes complex conjugation. In addition, the spectral expansion (9) identically satisfies the homogeneous Dirichlet-Neumann boundary conditions (6) since

$$
\Phi_{m}( \pm 1)=\Phi_{m}^{\prime}( \pm 1)=0
$$

the prime expressing derivation with respect to the wall-normal coordinate $\mathrm{d} / \mathrm{d} y$. Further detail on the spatio-temporal numerical discretisation of equation (7) may be found in Appendix A. 
We have used the 2-norm of the expansion coefficients vector a, as defined by

$$
\|\mathbf{a}\|=\left[\sum_{\ell=-L}^{L} \sum_{m=0}^{M}\left|a_{\ell m}\right|^{2}\right]^{1 / 2}
$$

to graphically represent the amplitude of solutions. Travelling wave solutions have a constant amplitude, while the instantaneous amplitude of time-dependent waves varies with time. In this latter case we have chosen to represent the maximum amplitude unless otherwise specified.

\section{SUB AND SUPERHARMONIC BIFURCATIONS OF TOLLMIEN- SCHLICHTING WAVES}

For sufficiently high Reynolds numbers, plane Poiseuille flow admits a multiplicity of solutions that coexist with the basic parabolic profile. The simplest such solutions arise directly from linear instability of the PPF base flow [29, 30]. They are relative equilibria, i.e. rigid structures that travel in the streamwise direction, and go by the name of TollmienSchlichting waves (TSW for short) [31,32]. They are characterized by a clear-cut streamwise periodicity of wavelength $\lambda_{0}=2 \pi / k_{0}$ (where $k_{0}$ is the fundamental wavenumber) and a travelling or phase speed c. For this particular type of solution, the Fourier coefficients in (9) read

$$
a_{\ell m}(t)=a_{\ell m}^{\mathrm{TW}} \mathrm{e}^{-\mathrm{i} \ell k_{0} c t}
$$

where $a_{\ell m}^{\mathrm{TW}}$ are time-independent Fourier-Legendre coefficients uniquely describing, except for an arbitrary initial phase, the wave solid-body structure. See Appendix B for a detailed descripton of the numerical methods employed in the computation and continuation of TSW.

Figure 1(a) shows a three-dimensional projection of the amplitude of Tsws, represented by the norm $\|\mathbf{a}\|$ introduced in (14), as a function of the streamwise wavenumber $k$ and the Reynolds number Re. This figure is to be compared with figure 4 in [33] for the constant pressure gradient formulation of the problem. Since in the present formulation all solutions are represented by their departure from the reference PPF parabolic base flow, this latter is uniquely represented by the $\|\mathbf{a}\|=0$ plane. The neutral stability curve of the PPF base flow is the parabola on this plane, whence the TSW family of solutions branches off. The 
(a)

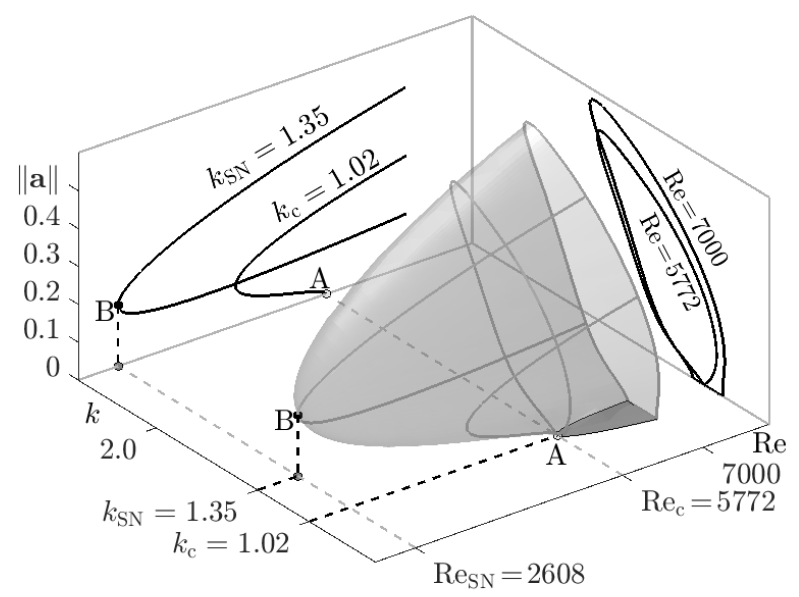

(b)

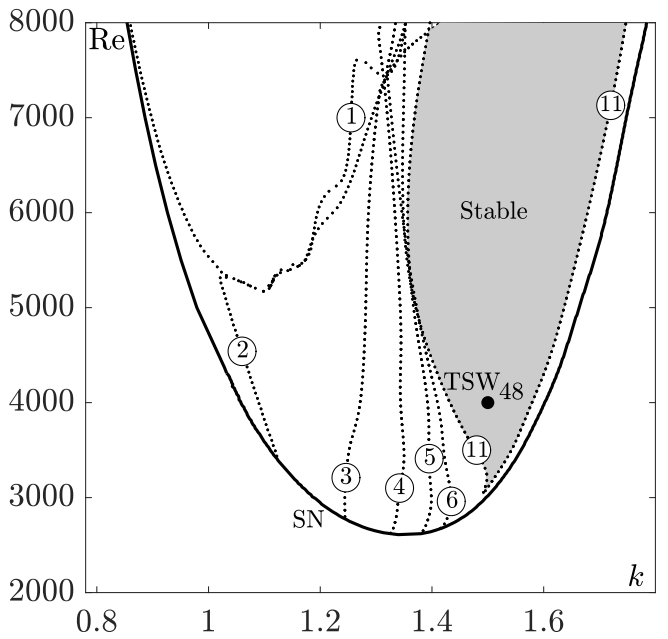

FIG. 1: (a) Tollmien-Schlichting waves (TSW) in parameter space for constant mass flow PPF. The projection depicts the norm $\|\mathbf{a}\|$ as a function of $(k, \operatorname{Re})$. (b) Subharmonic linear stability analysis of upper-branch TsWs. The gray area denotes linear stability of TsWs to all infinitesimal perturbations, both super- (1) and sub-harmonic (M, $n>1)$.

minimum Reynolds number point on the linear stability curve (labelled A), sets the wellknown critical pair $\left(k_{\mathrm{C}}, \mathrm{Re}_{\mathrm{C}}\right)=(1.02,5772.22)$, first computed in [30] in 1971. The area bound by the neutral curve (dark gray shadowed region) delimits the region of parameter space where PPF is linearly unstable. The bifurcation of TSWs is subcritical [31] for a certain range of wavenumbers around the critical $k_{\mathrm{C}}$, as clearly visible from the shape of the surface, which extends towards Reynolds numbers lower than $\operatorname{Re}_{\mathrm{C}}$. The existence of TSWs reaches as low as $\left(k_{\mathrm{SN}}, \operatorname{Re}_{\mathrm{SN}}\right) \approx(1.35,2608)$, where TSWs first arise at a saddle-node or fold bifurcation (labelled B). Subcriticality is remarkable in that $\operatorname{Re}_{S N}$ is significantly lower than $\operatorname{Re}_{\mathrm{C}}$, and also well below its pressure-driven counterpart $\operatorname{Re}_{\mathrm{SN}}^{\mathrm{P}} \approx 2939.03$ as reported in former studies [33, 34]. To guide the eye, figure 1(a) also depicts cross-sections of the three-dimensional surface representing the TSWs family for a couple of Re and $k$ values (gray curves on the Tsw surface) along with corresponding orthogonal projections on the cartesian planes (black solid curves). In the subcritical region around the saddle-node point $\left(k_{\mathrm{SN}}, \mathrm{Re}_{\mathrm{SN}}\right)$, two distinct TSW solutions simultaneously coexist with the base flow for the same values of the parameters. We call them the lower- and upper-branch solutions according to the compared value of their respective amplitudes. Lower-branch solutions are 
unstable, their unstable manifold pointing toward the base flow on one side and towards the upper-branch solution on the other. Upper-branch solutions are instead initially stable in a sufficiently close neighbourhood of the saddle-node point.

Numerous recent numerical studies have focused their attention on identifying spatiallymodulated waves that arise in open shear flows $[15,24,25]$. Linear stability analyses of constant pressure-gradient and constant mass-flux PPF reveal that upper-branch TSWs typically exhibit superharmonic Hopf bifurcations for Re moderately larger than $\operatorname{Re}_{\mathrm{SN}}[1,27,34,35]$. By superharmonic we mean that the Tsw becomes unstable with respect to perturbations of their same wavelength or shorter. This instability leads to branches of locally stable timeperiodic TSWs, hereafter called PTSW, that share wavelength with the TSW they bifurcate from. These coherent states are relative periodic orbits, since the emerged Hopf frequency bestows the wave a genuine time periodicity.

Systematic subharmonic linear stability analysis of PPF has been performed in the past [36] and more recently [1] in an attempt to cast light on streamwise localisation mechanisms of propagating waves. The stability of a wavetrain consisting of $n$ replicas of the same TSW $\left(\mathrm{R}_{n}\right)$ to perturbations of a wavelength that fits $m$ times in the replicated domain is taken into consideration. See Appendix B for technical details on the numerical implementation of the Arnoldi stability analysis tool. Figure 1(b) shows the regions in $(k, \mathrm{Re})$ parameter space where, according to Arnoldi linear stability analysis (B6), a Tsw of wavelength $\lambda=2 \pi / k$ exhibits subharmonic Hopf bifurcations with respect to perturbations of wavelength $n \lambda$, considering all values of $n \in \mathbb{N}$ up to 11 . The first superharmonic Hopf bifurcation occurs along the line labelled (1) in figure 1(b), such that TSWs are superharmonically unstable beyond this line. A train consisting of two TSWs $\left(\mathrm{R}_{2}\right)$ becomes unstable to time-dependent perturbations to the left of curve (2) which, simply put, means that the train $R_{2}$ is unstable in this region with respect to perturbations of length $\Lambda=2 \lambda_{0}$. The same goes for a train of three TSWs to the left of curve (3), and so on and so forth. The stability region of upper-branch TSWs shrinks as longer and longer perturbations are taken into consideration. However, the stability boundaries quickly saturate as $n$ increases, such that very little progression is observed for $n>11$ and beyond. Computations suggest that upper-branch Tsws may be linearly stable to all infinitesimal perturbations within a finite region of parameter space, liberally approximated here by the shaded area in figure 1(b). As a test, time-stepping at $\mathrm{Re}=4000$ of a $k=1.5 \mathrm{Tsw}$ replicated $n=48$ times (indicated by a bullet labelled $\mathrm{TSW}_{48}$ ) 
within a domain of length $\Lambda \approx 200 h$ did not result in destabilisation.

The subharmonic Hopf bifurcations described above were used by MM15 as the starting point for the study of localisation mechanisms of waves in long domains. In the case of the Swift-Hohenberg amplitude equation, the mechanism underlying the appearance of localised steady states follows the homoclinic snaking scenario [37, 38]. Homoclinic snaking has successfully provided an explanation for spanwise localisation of exact solutions in doubly extended shear flows such as plane Couette flow $[12,14]$. However, snaking is not the only possible mechanism leading to spanwise localisation. Alternative bifurcations, such as that exhibited by a spanwise-extended model of plane Couette flow [39], might also be at play. Homoclinic snaking, with modifications resulting from the broken $Z_{2}$ symmetry, was also explored by MM15 as a potential mechanism for localisation in the streamwise direction. Parametric continuation of localised travelling waves in both Reynolds number and domain wavelength concluded that although a mechanism analogous to snaking was possible, it is structurally unstable and very sensitive to small changes in wavelength. In addition, no evidence was found of ladder solutions, a constituent ingredient of homoclinic snaking, or of connections to the equivalent of antisymmetric solutions, which cannot exist in a system that does not possess the required symmetry.

\section{ASYMMETRIC TOLLMIEN-SCHLICHTING WAVES}

All streamwise-periodic solutions in PPF known so far preserve a remnant of the original streamwise-wall-normal $S O(2) \times Z_{2}$ symmetry of the problem in the form of a halfwavelength-streamwise-shift \& wall-normal-reflect symmetry

$$
\mathcal{S}:[u,-v ; p](x+\pi / k,-y ; t)=[u, v ; p](x, y ; t)
$$

Solutions breaking this symmetry might well lead to alternative localisation mechanisms biased to either one of the channel walls. We have set out to explore the eigenspectrum of $\mathcal{S}$ symmetric TSWs, obtained via Arnoldi linear stability analysis, in the search for $\mathcal{S}$-breaking pitchfork bifurcations. Eigenmodes have been systematically tracked and monitored for the TSW family of solutions throughout two-dimensional parameter space $(k, \operatorname{Re})$. Figure 2(a) shows two such pitchfork bifurcations ( $\mathrm{P}_{1}$ and $\mathrm{P}_{2}$, indicated with triangles) on a continuation curve of Tsws (gray line) at Re $=10^{4}$. At this Reynolds number, Tsws are linearly stable 
(a)

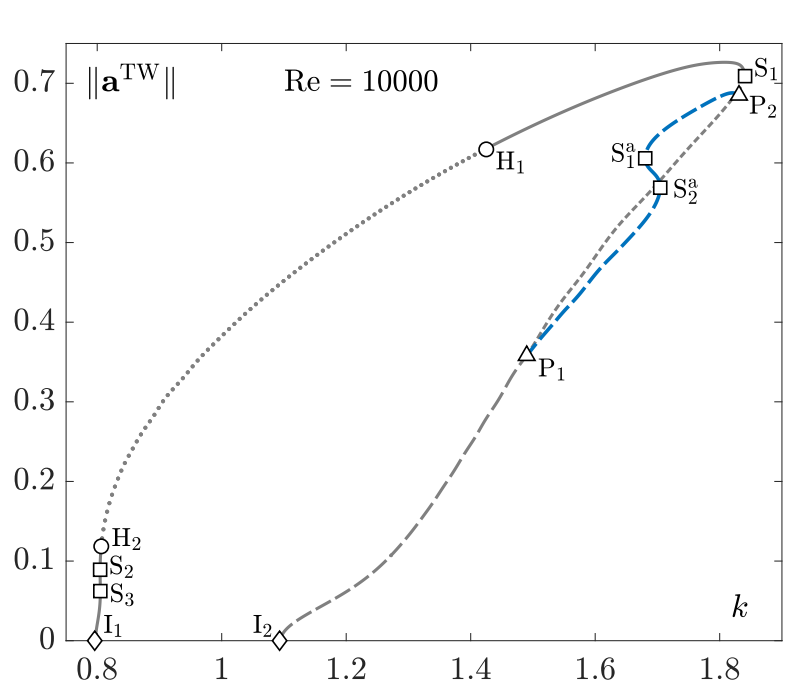

(b)

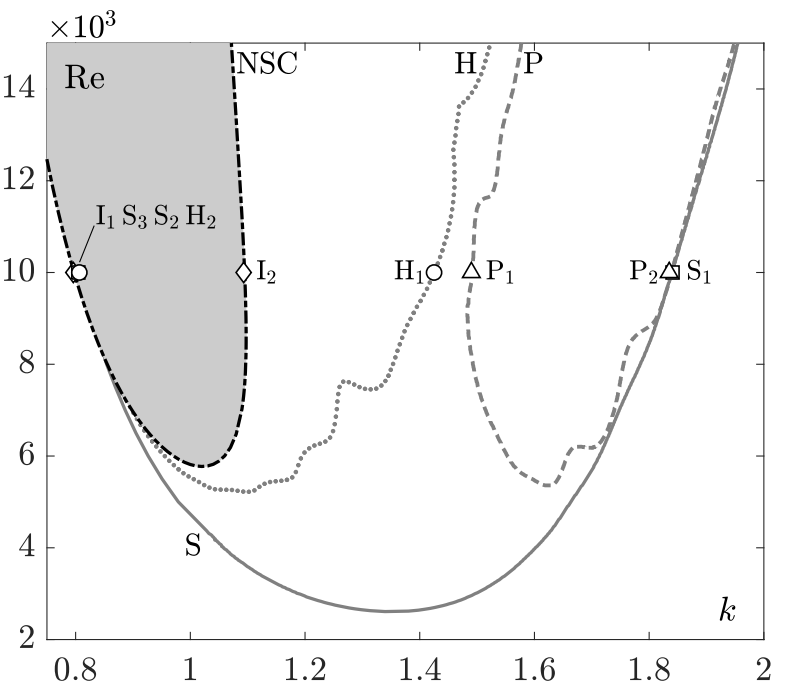

FIG. 2: (a) Pseudo-arclength wavelength $k$-continuation of Tsw (gray) and ATsw (blue) TollmienSchlichting waves for $\mathrm{Re}=10^{4}$. Symbols indicate saddle-node (squares, s), Hopf (circles, H), and pitchfork (triangles, P) bifurcations. Also shown are the streamwise-invariance-breaking bifurcation points (diamonds, I) whence TSWs branch off the base flow. Line styles denote branches with different stability properties: stable (solid), one unstable complex pair (dotted), one unstable real (long-dashed) or two unstable real (short-dashed) eigenmodes. (b) Bifurcation diagram of TSWs. Shown are saddle-node (s, solid gray line), superharmonic Hopf (H, dotted) and pitchfork (P, dashed) bifurcation curves. The gray shaded area indicates the region where PPF is linearly unstable, bound by the neutral stability curve (NSC, I, dash-dotted).

(solid) in three separate regions, respectively bounded by points $\mathrm{S}_{1}-\mathrm{H}_{1}, \mathrm{H}_{2}-\mathrm{S}_{2}$, and $\mathrm{S}_{3}-\mathrm{I}_{1}$. In between Hopf bifurcation points $\mathrm{H}_{1}$ and $\mathrm{H}_{2}$, TSWs possess one unstable complex pair (dotted) that leads to PTSWs as described by MM15. The branch sector delimited at either end by saddle-node points $\mathrm{S}_{2}$ and $\mathrm{S}_{3}$ is unstable with one positive real eigenvalue (long-dashed), as also are TSWs in the segments $\mathrm{I}_{2}-\mathrm{P}_{1}$ and $\mathrm{P}_{2}-\mathrm{S}_{1}$. The associated eigenmode is $\mathcal{S}$-preserving and the unstable manifold of these lower-branch Tsws lead either toward upper-branch TSWs to one side and either base flow or another upper-branch of TSWs to the other. TSWs incorporate a second positive real eigenvalue with $\mathcal{S}$-breaking associated eigenmode along the segment $\mathrm{P}_{1}-\mathrm{P}_{2}$ (short-dashed). The two pitchfork bifurcation points, which happen to be supercritical, are connected by a bridging branch of asymmetric TSWs, or ATSWs (blue 
line), that inherits the unstable real eigenmode from lower-branch TSWs. The presence of a pair of saddle-node bifurcations along the branch stabilizes the unstable eigenmode, if only briefly, thus leaving a small region of stability (solid) between $\mathrm{S}_{1}^{\mathrm{a}}$ and $\mathrm{S}_{2}^{\mathrm{a}}$. The bifurcation points of figure 2(a) unfold into bifurcation curves when tracked in $(k, \mathrm{Re})$ parameter space. Figure 2(b) depicts the loci of pitchfork bifurcations of lower-branch TSWs (P, gray dashed line), along with the upper-branch Hopf (H, dotted, (1) in figure 1b) and the saddle-node ( $\mathrm{s}$, solid) bifurcation curves. The lower- $k$ portion of the saddle-node bifurcation curve has split in two by the time it reaches $\operatorname{Re}=10000$, but this is barely noticeable in the figure. The pitchfork bifurcation curve reaches as low as $\mathrm{Re} \approx 5350<\mathrm{Re}_{\mathrm{c}}$ which renders ATSWs slightly subcritical in the sense that they coexist with the stable base flow, whose linear instability region is shaded with gray (bound by the neutral stability curve NSC -black solid-, corresponding to an axial-invariance-breaking I of the base flow) in figure 2(b).

Figures 3(a) and (b) show streamfunction perturbation colourmaps of the lower-branch TSW and the ATSW, respectively, at $(k, \operatorname{Re})=\left(1.7,10^{4}\right)$, in a close neighbourhood of the pitchfork bifurcation point $\mathrm{P}_{1}$. The colour range has been set equispaced, symmetric and equal for both waves in order to adequately convey the effects of the $\mathcal{S}$-symmetry-breaking. In terms of streamfunction perturbation, the $\mathcal{S}$ symmetry reads

$$
\mathcal{S}: \widetilde{\Psi}_{\mathrm{TSW}}(x, y ; t)=-\widetilde{\Psi}_{\mathrm{TSW}}\left(x+\frac{\pi}{k},-y ; t\right)
$$

so that $\mathcal{S}$-symmetric solutions change sign of $\tilde{\Psi}$ under a half-wavelength shift followed by reflection across the horizontal midplane. While the TSW clearly preserves the $\mathcal{S}$ symmetry, the ATsw has slightly but obviously disrupted it. The symmetry-breaking has been qualitatively assessed by subtracting one wave from the other in figure $3(\mathrm{~d})$. Given that the waves are close to the bifurcation point, the difference between the two is closely aligned with the bifurcating eigenmode, which is shown in figure $3(\mathrm{c})$.

The effects of $\mathcal{S}$ on the Fourier-Legendre spectrum of solutions and eigenmodes allows straightforward detection of the symmetry. Formal substitution of the spectral expansion (9) into identity (17) leads to

$$
\sum_{\ell, m}^{L, M} a_{\ell m} \mathrm{e}^{\mathrm{i} \ell k x} \Phi_{m}(y)=\sum_{\ell, m}^{L, M}(-1)^{\ell+m+1} a_{\ell m} \mathrm{e}^{\mathrm{i} \ell k x} \Phi_{m}(y),
$$

where we have used the parity rule of Legendre polynomials $\mathrm{L}_{m}(-y)=(-1)^{m} \mathrm{~L}_{m}(y)$. According to (18), non-vanishing Fourier-Legendre spectral coefficients corresponding to a TSW 
(a)

$\widetilde{\Psi}_{\text {TSW }}$
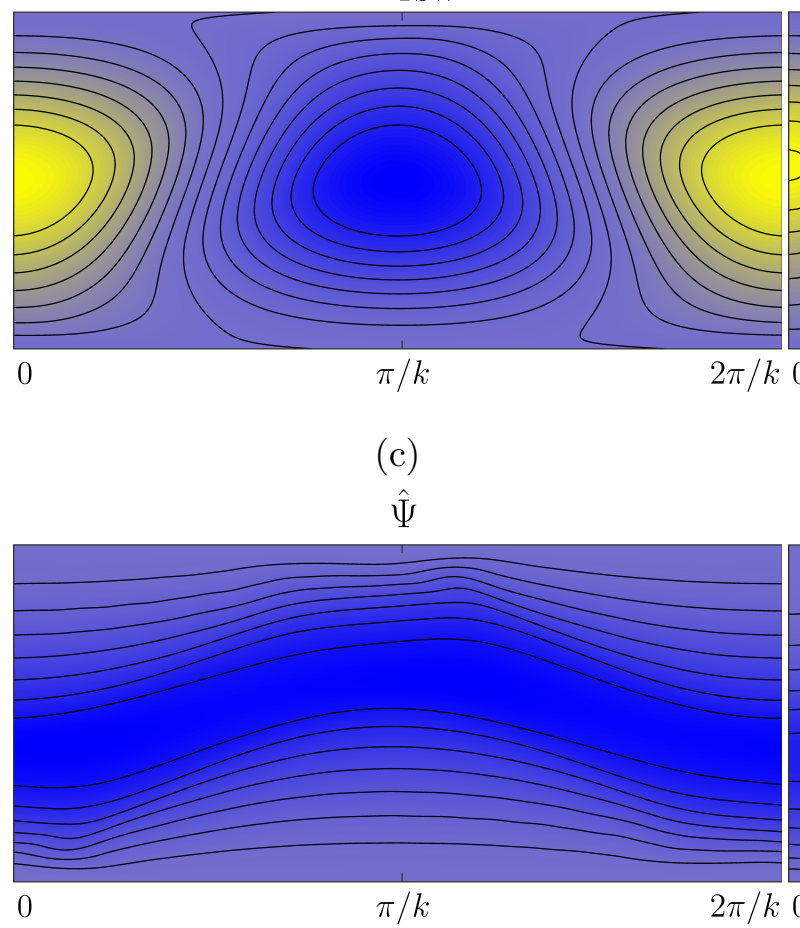

(c)

$\hat{\Psi}$

0

$(\mathrm{c})$ (b)

$\widetilde{\Psi}_{\text {ATSW }}$

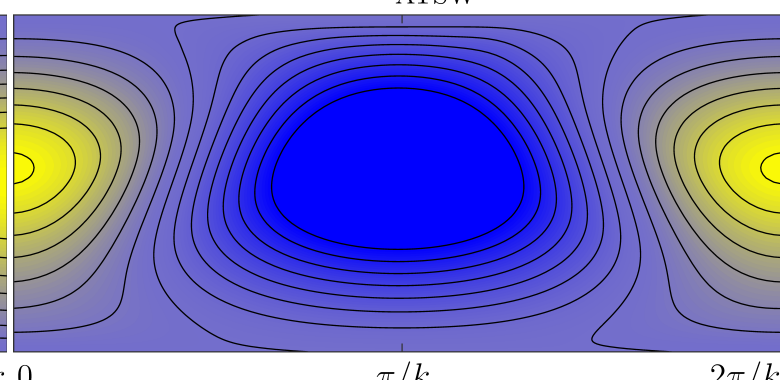

(d)

$\widetilde{\Psi}_{\mathrm{ATSW}}-\widetilde{\Psi}_{\mathrm{TSW}}$

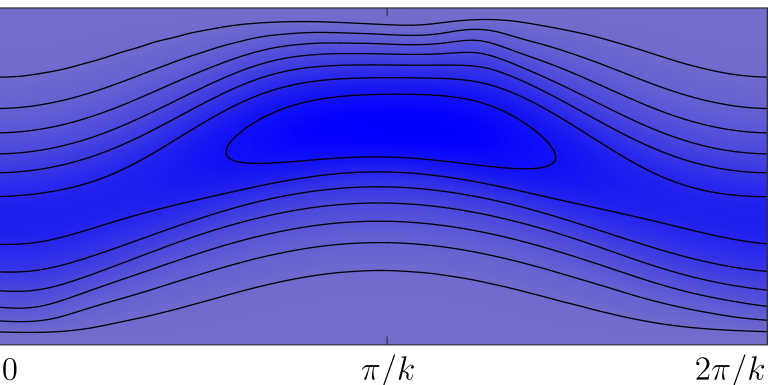

FIG. 3: Streamfunction perturbation $\tilde{\Psi}$ of travelling wave solutions in the vicinity of the pitchfork bifurcation point $\mathrm{P}_{1}$ of figure 2 for $(k, \operatorname{Re})=\left(1.7,10^{4}\right)$. (a) TSW and (b) ATSW. Contour levels are equispaced in the interval $\tilde{\Psi} \in[-0.14,0.14]$ with step $\Delta \tilde{\Psi}=0.02$. (c) Bifurcating eigenmode $\hat{\Psi}$. (d) Difference $\tilde{\Psi}_{\mathrm{ATSW}}-\tilde{\Psi}_{\mathrm{TSW}}$ between TSW and ATSW.

must have $(\ell, m)$ pairs satisfying the selection rule

$$
\ell+m=2 n+1,
$$

for $n \in \mathbb{Z}$. The presence of the $\mathcal{S}$ symmetry can therefore be ascertained by verifying that all coefficients not following (19) vanish exactly.

Arnoldi stability analysis applied to the eigenproblem (B6) provides the unstable expansion-coefficients-eigenvector associated with the pitchfork bifurcation $\left\{\varepsilon_{\ell m}\right\}$. The corresponding eigenmode streamfunction perturbation fields are given by

$$
\hat{\Psi}(x, y)=\sum_{\ell=-L}^{L} \sum_{m=0}^{M} \varepsilon_{\ell m} \Phi_{m}(y) .
$$

Eigenmodes breaking the $\mathcal{S}$ symmetry ought to be $\mathcal{S}$-antisymmetric

$$
\mathcal{A}: \hat{\Psi}(x, y)=\hat{\Psi}\left(x+\frac{\pi}{k},-y\right),
$$


as a visual inspection of the eigenmode in figure 3(c) clearly illustrates. By virtue of (21), the eigenmode expansion (20) must satisfy

$$
\sum_{\ell, m}^{L, M} \varepsilon_{\ell m} \mathrm{e}^{\mathrm{i} \ell k x} \Phi_{m}(y)=\sum_{\ell, m}^{L, M}(-1)^{\ell+m} \varepsilon_{\ell m} \mathrm{e}^{\mathrm{i} \ell k x} \Phi_{m}(y),
$$

so that non-vanishing $(\ell, m)$ Fourier-Legendre pairs of an $\mathcal{S}$-symmetry-breaking eigenmode follow instead an even selection rule of the form

$$
\ell+m=2 n
$$

with $n \in \mathbb{Z}$. All coefficients other than those in accordance with (23) must be identically zero. Selection rules (19) and (23) have been confirmed numerically by direct examination of the sparsity structure of the coefficients $a_{\ell m}$ of TSWs and $\varepsilon_{\ell m}$ of $\mathcal{S}$-symmetry-breaking eigenmodes.

In order to clearly identify symmetry-broken solutions and to properly quantify the degree to which the symmetry has been disrupted we have defined an appropriate symmetry parameter as

$$
S=\sum_{j=0}^{L-1} \omega(\Lambda j / L, 0)
$$

where $\omega(x, 0)$ is the flow vorticity evaluated at any point $(x, 0)$ along the channel mid line $y=0$. The parameter $S$ is therefore obtained as the sum along the channel mid plane of the vorticity values at the equispaced Fourier streamwise nodes $x_{j}=\Lambda j / L$, for $j=0,1, \ldots, L-1$. Since a TSW respects the half-period-shift \& reflect symmetry, vorticity exactly changes sign between two points on the centre line located exactly half a wavelength apart, so that the sum along the centre line yields $S=0$. In the meantime, an ATSW breaks the symmetry, which leads to an imbalance between vortices in the upper and lower half of the domain that generally produces instead a net sum $S \neq 0$. As is the case for any pitchfork bifurcation, the ATSWs appear in conjugate-symmetric pairs, such that two solution branches exist simultaneously which are mutually related by the $\mathcal{S}$ symmetry. The symmetry parameter $S$ associated with the TSW and the bifurcated ATsw is represented in figure 4(a) as a function of the Reynolds number, for three different values of $k$. The pitchfork bifurcation (triangle) is supercritical for most $k$ but becomes slightly subcritical within certain ranges of $k$. Nevertheless, the subcritical branches turn in a saddle-node 
(a)

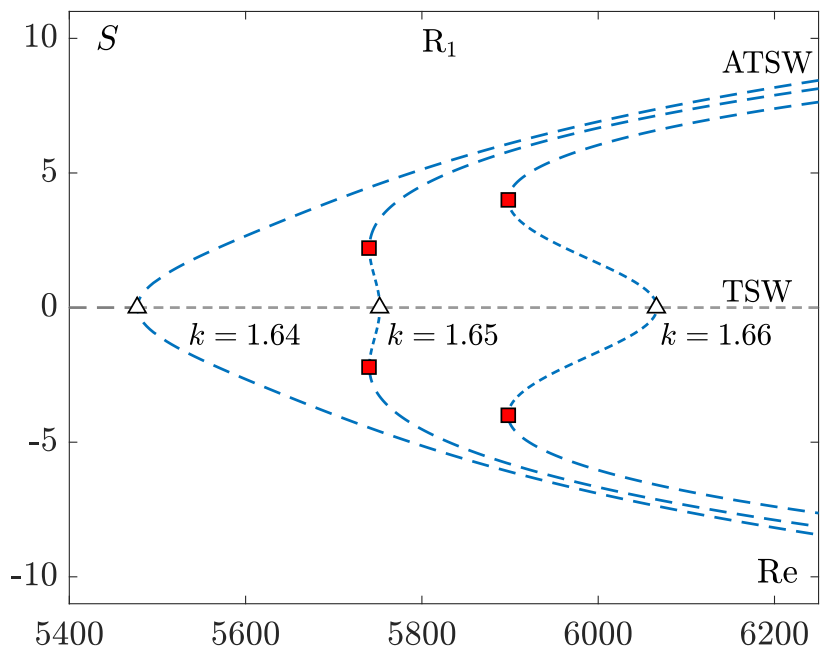

(b)

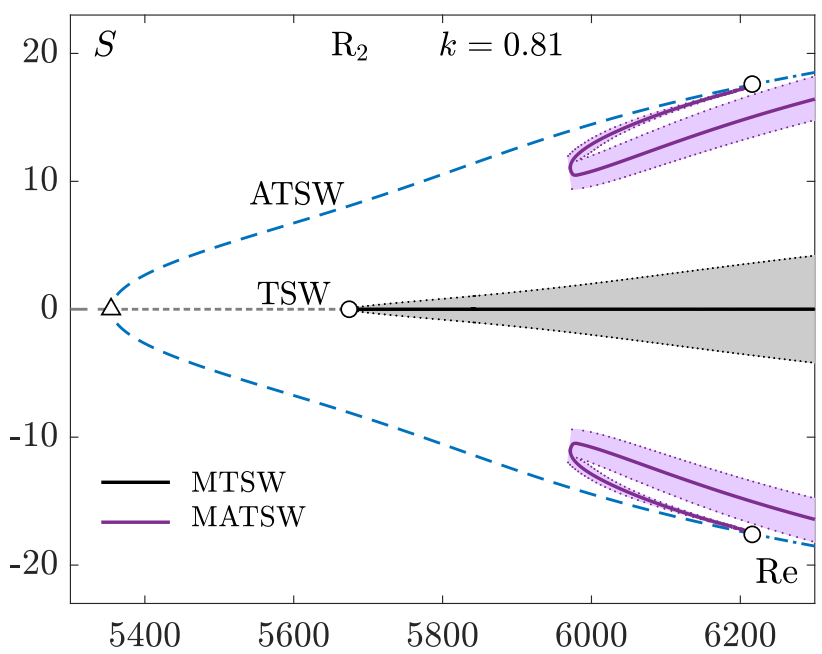

FIG. 4: Symmetry parameter $S$ of TSW and ATSW solution branches as a function of Re for (a) small domains of fundamental wavenumber $k=1.64,1.65$ and 1.66 (see labels) fitting a single wavelength, and (b) a larger domain of wavenumber $k=0.81$ accommodating an $\mathrm{R}_{2}$-replicated wave. Symbols mark pitchfork (triangles), saddle-node (squares, red to indicate correspondence to the subcritical saddle-nodes in figure 5a) and subharmonic Hopf bifurcations (circles). TSw and ATSW linestyles as for figure 2. The time-average (solid line) and oscillation amplitude (delimited by the shaded region) of MTSws (black) and MATsws (violet) are also indicated.

bifurcation (red squares), such that they always finally extend towards increasing values of Re. Figure 5(a), which zooms in $(k, \mathrm{Re})$ parameter space on the pitchfork bifurcation curve of figure 2(b), shows the loci of ATsws saddle-node bifurcations (red line). There exist two separate parameter regions where ATsws are subcritical, and then only very mildly.

All ATsws identified in our explorations have been found to emerge from the lower branch of ordinary TSWs, and within a range of streamwise wavenumbers $k$ for which the latter do not bifurcate from the base Poiseuille flow. We have no explanation as to why all symmetrybreaking pitchfork bifurcations should occur on the lower branch of TSWs but, as it happens, upper branch solutions seem to require the onset of temporal chaos to fully disrupt the last remnants of the reflectional symmetry about the channel centre-line. Figure 5(b) summarizes the comprehensive exploration performed in the present study by depicting the bifurcated ATSWs (colour lines) emerging from the lower branch of TSWs (gray). The continuations have been performed for streamwise wavenumbers ranging within the interval 
(a)

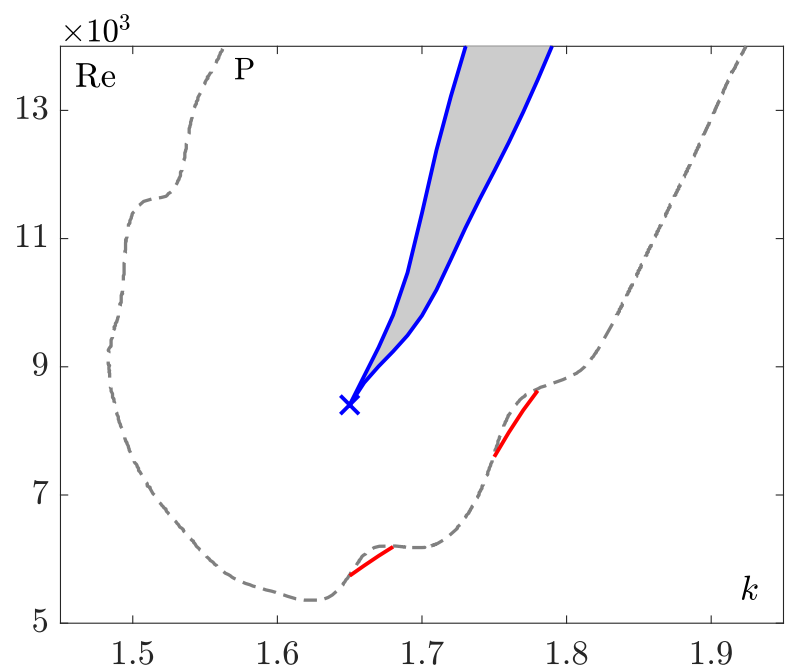

(b)

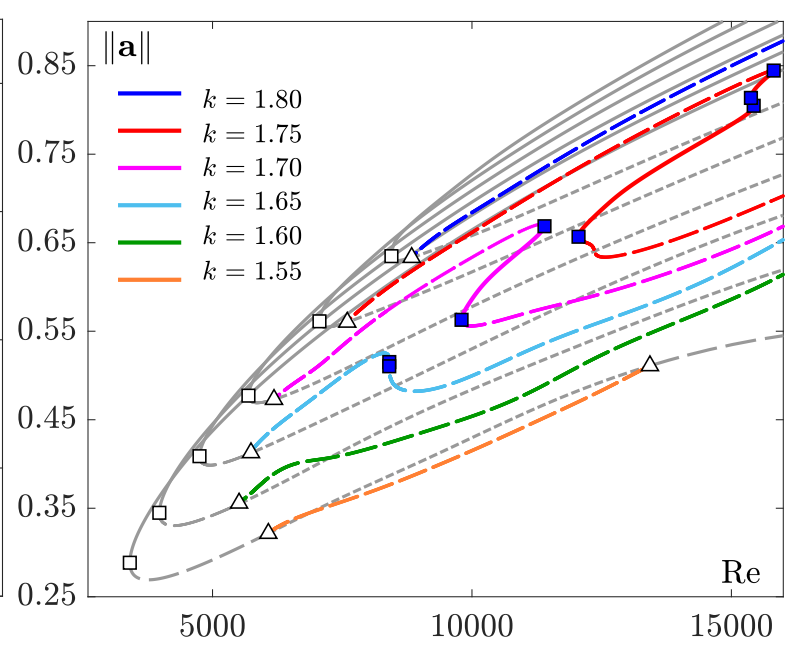

FIG. 5: (a) Lower-branch TSWs bifurcation diagram in $(k, \mathrm{Re})$ parameter space. Shown are the pitchfork bifurcation curve for TSWs (dashed gray), the occasional saddle-node bifurcation curve of subcritical ATSWs (red) and the two saddle-node bifurcation curves of ATSWs (blue) that produce a region of linear stability (shaded area). The cross sign indicates a cusp bifurcation point at $(k, \operatorname{Re}) \simeq(1.65,8406)$. (b) $\|\mathbf{a}\|$ of TSWs (gray lines) and ATsWs (colour lines) as a function of Re and $k$. Line styles and symbols as for figure 2(a).

$k \in[1.55,1.80]$. Although ATSWs are initially linearly unstable at bifurcation, they eventually -yet briefly- become stable at sufficiently large values of $k$ and Re. As an example, the ATSW branch at $k=1.7$ inherits the unstable real eigenvalue as it bifurcates supercritically from TSWs at $\mathrm{Re} \approx 6430$ (triangle). The branch becomes stable, however, in a saddle-node bifurcation (blue square) at $\mathrm{Re} \approx 11388$ and extends towards decreasing Reynolds number until undergoing a second saddle-node bifurcation at $\mathrm{Re} \approx 9800$ that destabilises it anew. From this point on, the branch progresses again, unstable, in the direction of increasing Reynolds numbers. The double saddle-node bounds a region in $(k, \mathrm{Re})$ parameter space (gray shaded area in figure 5(a) bound by blue lines) where ATsws are stable. The lowest Re at which stable ATsws may be found is given by the cusp point (cross symbol) where the two saddle-node bifurcation curves (blue) intersect. 


\section{SPACE-MODULATED ASYMMETRIC TOLLMIEN-SCHLICHTING WAVES}

Hopf bifurcations (super or subharmonic) of Tsws introduce a time-periodicity on top of the mere travelling motion. This modulation can be understood as a genuine periodic orbit on the co-moving reference frame of the TSW. In this sense, the resulting Navier-Stokes solution is a time-periodic travelling wave (a relative periodic orbit). Solutions of this type are often unstable so they cannot be computed by simply time-stepping the system (A7) using the scheme (A8). These solutions can instead be computed and tracked in parameter space, regardless of their stability, by means of a Newton-Krylov-Poincaré method as that described in Appendix C.

The presence of subharmonic Hopf bifurcations on the lower branch of Tsws was already explored in detail by MM15. In some cases, this type of bifurcation leads to a time-periodic space-modulated Tollmien-Schlichting wave or MTSW that does not produce streamwise localisation. Figure 4(b) shows a branch of MTSWs (black solid) bifurcated subharmonically in a domain with fundamental wavenumber $k=0.81$ accommodating an $\mathrm{R}_{2}$-replicated $\mathrm{TSW}$. The MTSW clearly preserves, on average, the $\mathcal{S}$-symmetry as the identically zero value of the time-averaged $S$ parameter (solid line) reveals. The amplitude of the oscillation (gray shaded region), though, clearly shows that the symmetry is instantaneously broken. As a matter of fact, the $\mathcal{S}$-symmetry is traded for an equivalent space-time symmetry that renders the solution invariant under half a period evolution followed by reflection about the mid plane and an appropriate streamwise shift. Wavelength continuation confirms that these solution branches are created and annihilated at either end without the incorporation (or suppression) of an additional TSW replica. This is illustrated in figure 6 (a) for Re $=8000$, where a branch of modulated solutions (solid black curve) connects the two subharmonic Hopf points $\mathrm{H}_{21}$ and $\mathrm{H}_{22}$ (circles) on the lower branch of a twice-replicated $\mathrm{R}_{2}$ TSW branch. The role of these self-connecting non-localised modulated waves was not satisfactorily addressed by MM15. This will be the object of next section. Here we set out to clarify whether spacemodulation may also arise from ATsws and, if so, whether the resulting space-modulated ATSWs (MATSWs) may bear some connection with known MTSWs or evolve instead into localisation following a different mechanism.

Figure 4(b) shows how two mutually-symmetric branches of MATSws (violet) do indeed bifurcate from either branch of ATSWs at subcritical subharmonic Hopf points. In this case, 
(a)

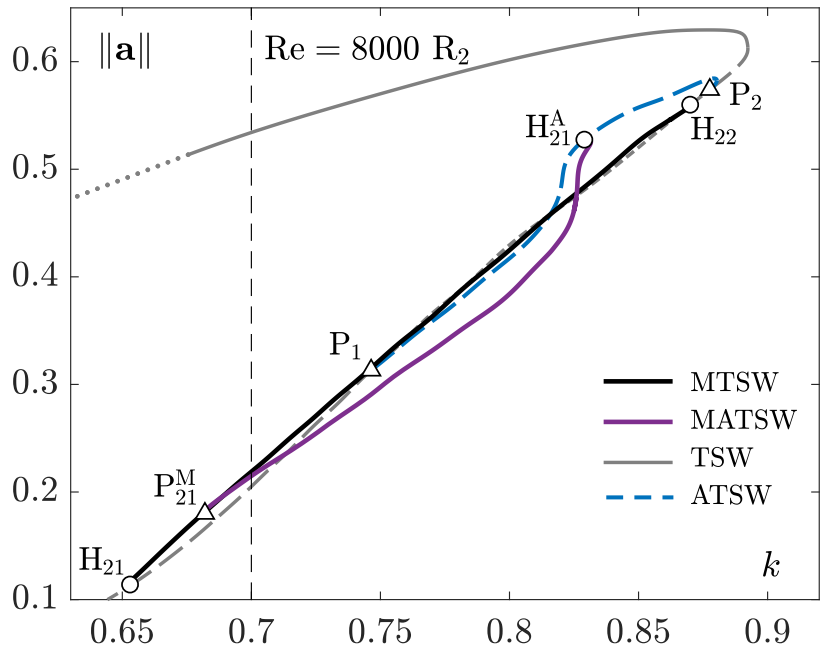

(b)

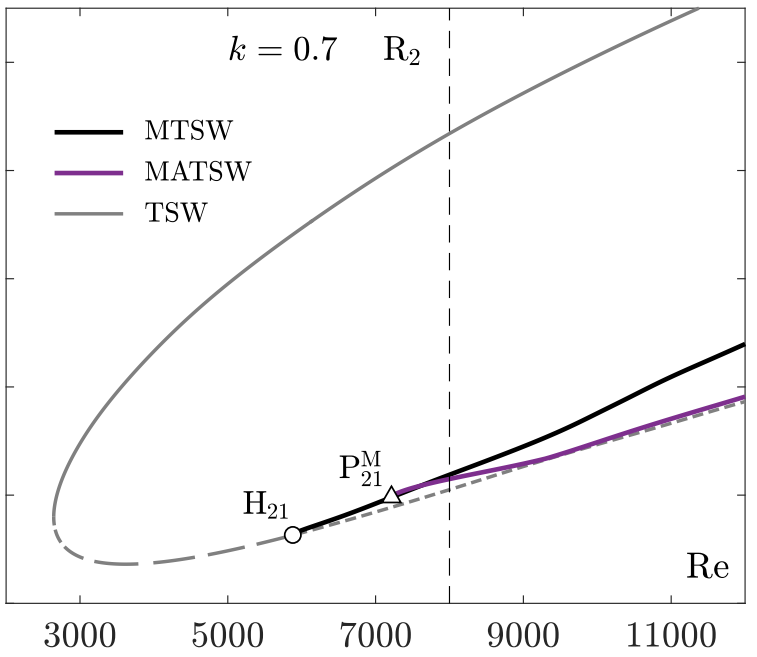

FIG. 6: (a) Wavelength $k$ - (at $\mathrm{Re}=8000)$ and (b) Re-continuation (at $k=0.7)$ of $\mathrm{R}_{2}$-replicated TSWs. Shown are TSWs (gray lines) along with bifurcating ATSW (blue), MTSW (black) and MATSW (violet). Hopf (circles, $\mathrm{H}_{2 i}$ ) and pitchfork (triangles, $\mathrm{P}_{2 i}$ ) $\mathrm{R}_{2}$-subharmonic bifurcation points are duly marked. Superscripts M and A denote bifurcation points on MTSW and ATSW branches, respectively. TSW linestyles as for figure 2 .

both the time-averaged value of the $S$ parameter (violet solid line) and the instantaneous value as expressed by the amplitude (pink shaded region) are different from zero, but the two separate branches are clearly symmetric to one another. We have performed a comprehensive subharmonic stability analysis along the continuation branches of ATSWs and found out that they also exhibit subharmonic Hopf bifurcations that generate branches of MATsws, even in short domains fitting just two fundamental wavelengths of the ATSW. Figure 6(a) shows a wavelength continuation of an ATSW (blue line) bifurcated from lower-branch Tsws (gray line) at $\mathrm{Re}=8000$. The ATSW branch connects two pitchfork bifurcation points $\left(\mathrm{P}_{1}\right.$ and $\mathrm{P}_{2}$, triangles) on the Tsw branch. The branch undergoes a subharmonic Hopf bifurcation $\left(\mathrm{H}_{21}^{\mathrm{A}}\right.$, circle), whence a new family of time-periodic space-modulated asymmetric waves MATSW (violet line) is issued. This MATsw branch is unstable, although stable MATsw have also been identified at much higher Reynolds numbers. Wavelength continuation of the MATSW branch reveals that it reconnects with the branch of MTSWs (black line), presumably at a pitchfork-of-cycles $\left(\mathrm{P}_{21}^{\mathrm{M}}\right.$, triangle). Floquet-Arnoldi linear stability analysis of the Poincaré first return map $(\mathrm{C} 1)$ corresponding to the relative periodic orbits associated with time- 
periodic waves has been employed to confirm the existence of this pitchfork bifurcation. Eigenvalues of the Poincaré map (Floquet exponents of the associated periodic orbit) have been accurately computed and monitored along the modulated solution branches. Analogous to the emergence of ATSWs from TSW, the Floquet stability analysis confirms that the MATSW branch is the result of a pitchfork bifurcation along the MTSw branch. Figure 6(b) shows the Reynolds continuation of both symmetric and asymmetric time-periodic modulated waves for $k=0.7$. Within the explored range of parameters, the MATSW branch emerges always from a supercritical pitchfork bifurcation on the MTSw branch and does in no case reconnect with any other know solution branch. This is consistent with the exploration of ATsws as summarized in figure 5(a), where these waves are shown non-existent for $k<1.48$ and $\operatorname{Re}<14000$. Consequently, since the MATSw solutions shown in figure 6 (b) have $k=0.7$, which corresponds to $\mathrm{R}_{2}$-subharmonic bifurcation from an original ATSW with $k=1.4$, reconnection cannot occur for this wavelength.

We have undertaken subharmonic stability analysis for $R_{3}, R_{4}$ and $R_{5}$ replicated ATSWs. Within the explored ranges of Reynolds numbers and wavelengths, we have not identified any remarkable bifurcation qualitatively different from the one that is already observed for $R_{2}$. Floquet stability analysis of the bifurcated MATSWs has also been carried out in replicated domains. In this case, subharmonic bifurcations have only been detected for four-fold $\mathrm{R}_{4^{-}}$ replicated MATSWs and beyond. Tracking MATsw branches in the long streamwise domains required for effective localisation poses an insurmountable challenge to available numerical resources on account of the large amount of unstable eigenmodes present. Besides, the relevance of highly unstable waves, however localised, to actual flow dynamics is at the very least questionable on the same grounds.

\section{INTERACTIONS AMONG THE VARIOUS MTSW SOLUTION BRANCHES}

In sufficiently long domains, stable localised time-periodic waves may exist in PPF for Reynolds numbers as low as $\operatorname{Re}=2330$ [40]. This type of solutions appear as a result of saddle-node bifurcations of relative periodic orbits upon increasing the Reynolds number, as parametric continuations by MM15 show [1, figure 2a]. Upper-branch states are stable within the range $\operatorname{Re} \in[2330,2615]$, and are the only localised stable solutions of the two-dimensional Navier-Stokes equations in extended domains known to date. For Re $>2615$, and sufficiently 
long channels, localised solutions start interacting with the classical TSWs. As a matter of fact, upper-branch localised states ultimately originate from subharmonic Hopf bifurcations occurring along the superharmonically-stable upper branch of $\mathrm{R}_{n}$-replicated Tsws, for $n \geq 3$. Within the range of Reynolds numbers explored in MM15, continuation to larger domains of MTSWs emerging from upper-branch $\mathrm{R}_{n}$ TSWs results in three different branch types. For sufficiently low Re, the MTsw branch bifurcated from $\mathrm{R}_{n}$ extends to arbitrarily long domains as the solutions become strongly localised and exhibit exponential decay at both the trailing and leading fronts [26]. Coexisting with this branch at the same Re, another branch of MTSWs connects subharmonic Hopf bifurcations on the upper and lower branch of $\mathrm{R}_{n+1}$ TSWs. At somewhat higher Re, wavelength continuation of the MTSW branch bifurcated from upper-branch $\mathrm{R}_{n}$ TSWs results in the development of a wave defect, becomes unstable in a saddle-node, and eventually reconnects with the unstable lower branch of $\mathrm{R}_{n+1}$ TSWs having added a new wave replica along the way. This latter branch is in fact the result of a codimension-2 bifurcation of the former two solution branches. At the double-critical point, another branch of the localising type, but this time starting from $\mathrm{R}_{n+1}$ upper-branch TsWs is created. At still higher Re and starting from long-wavelength subharmonic Hopf bifurcations, $k$-continuation of MTSWs occasionally produces exceedingly convoluted branches, so much so that neither extension to long domains nor reconnection to TSW branches has been found [1, figure 4a]. The role and fate of these never-ending, intricate, MTSw branches remains an open question.

A completely different type of MTSw branch was also found by MM15 that uneventfully connects pairs of subharmonic Hopf bifurcation points on the higher- $k$ end of the same branch of $\mathrm{R}_{n}$-replicated TSWs. These other MTSW solutions did not seem to interact with any of the aforementioned MTSw branches, these latter all interrelated by the bicritical point and intimately associated to the streamwise localisation mechanism under scrutiny, and where therefore deliberately overlooked by MM15.

We focus here on these seemingly unrelated MTSW branches and explore whether they may lead to a localisation mechanism different from the one already described. We have systematically monitored all subharmonic bifurcations undergone by lower-branch Tsws and tracked the MTSW branches issued from them for increasing values of the Reynolds number. Figure 7(a) shows the two MTSw solution branches (black lines) emerged from the only two subharmonic Hopf bifurcations (circles, $\mathrm{H}_{41}$ and $\mathrm{H}_{42}$ ) of lower-branch TsWs that exist in an 
(a)

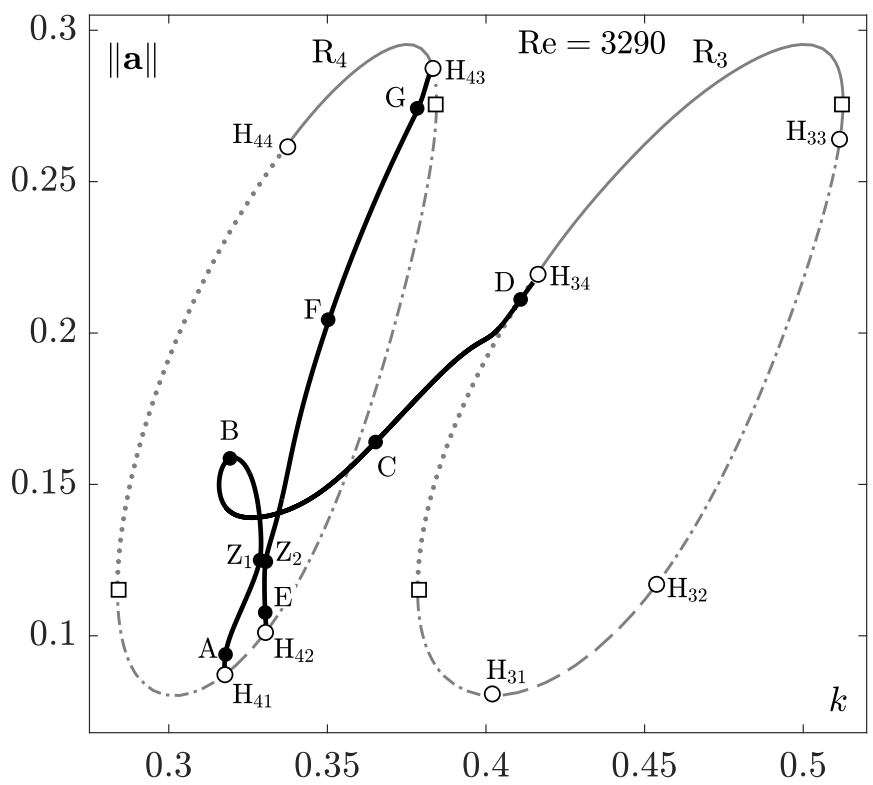

(b)

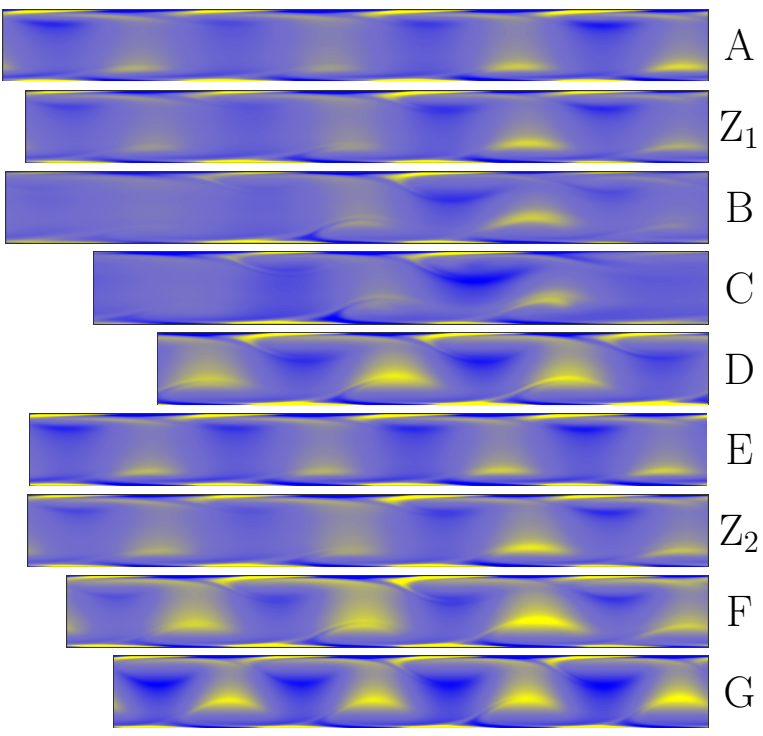

FIG. 7: MTSW branches (black lines) issued from $\mathrm{R}_{4}$-subharmonic Hopf bifurcations of lower-branch TSWs (gray lines) at $\operatorname{Re}=3290$. (a) Amplitude $\|\mathbf{a}\|$ vs wavenumber $k$. Symbols denote saddle-node (squares) and Hopf (white circles, $\mathrm{H}_{4 i}$ ) bifurcations. Instability to one real and one complex pair of eigenmodes is indicated with a dash-dotted line. Rest Tsw-related linestyles as for figure 2. (b) Vorticity $\omega$ colourmaps of selected MTSW solutions duly marked (black circles) and labelled in (a).

$\mathrm{R}_{4}$-replicated domain at $\mathrm{Re}=3290$. A few vorticity colourmaps of snapshots taken along the continuation branches (black circles with labels) have been represented in figure 7(b) for illustration purposes. The branch starting at the Hopf bifurcation $\mathrm{H}_{41}$ closely resembles an $\mathrm{R}_{4}$ train of lower-branch TSWs at point $\mathrm{A}$. By the time the branch reaches point $\mathrm{z}_{1}$, the spatial modulation is clear and a defect in the TSW pattern has emerged. The branch goes in a loop as the solution trades one TSW replica for a laminar gap (B) and finally extends to higher $k$ progressively closing the gap through $\mathrm{C}$ to $\mathrm{D}$, where something close to an $\mathrm{R}_{3}$ TSW train fills the fundamental periodic domain before landing on the upper branch of TSWs at Hopf point $\mathrm{H}_{34}$. Meanwhile, the MTSW family branching off TSWs at the Hopf point $\mathrm{H}_{42}$ is one of these hitherto apparently irrelevant branches uneventfully bridging subharmonic Hopf bifurcations on the same $\mathrm{R}_{n}$-replicated Tsw branch, this time ending at Hopf point $\mathrm{H}_{43}$. Vorticity colourmaps show a barely imperceptibly imperfect $R_{4}$ train of lower-branch Tsws close to $\mathrm{H}_{42}$ at point $\mathrm{E}$. The solution has clearly developed a defect by the time it reaches 
(a)

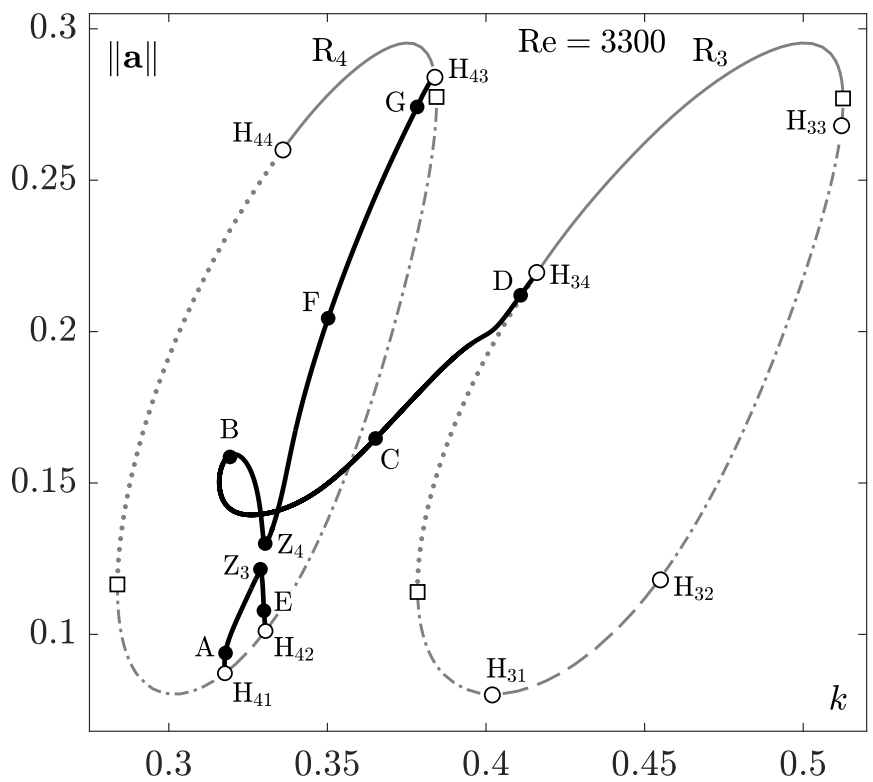

(b)

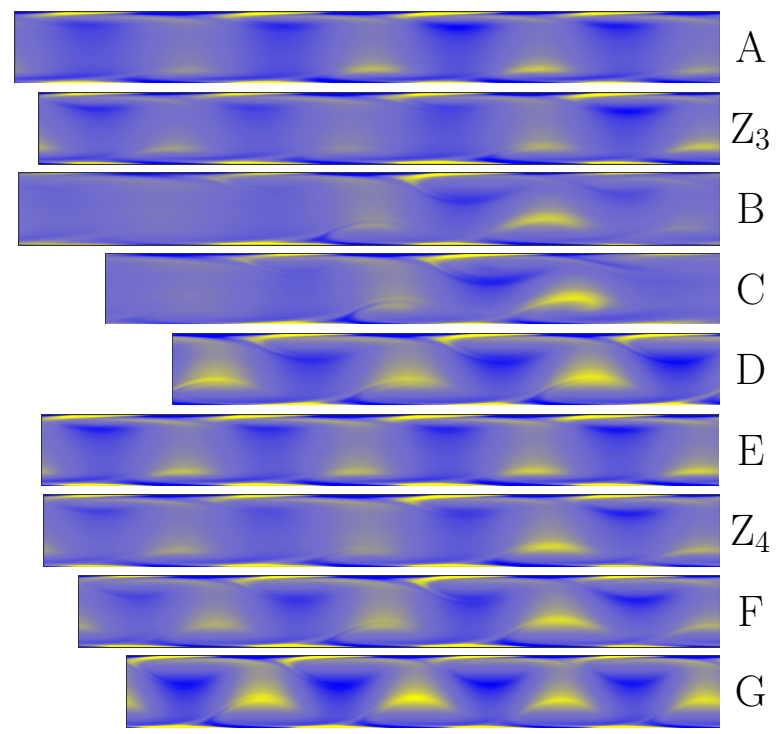

FIG. 8: MTSW branches issued from $\mathrm{R}_{4}$-subharmonic Hopf bifurcations of lower-branch TSWs at $\operatorname{Re}=3300$. (a) Amplitude $\|\mathbf{a}\|$ vs wavenumber $k$. (b) Vorticity $\omega$ colourmaps of selected MTsw solutions. Symbols and linestyles as for figure 7.

point $\mathrm{Z}_{2}$ that persists through point $\mathrm{F}$ but is later gradually attenuated until recovering the $\mathrm{R}_{4}$ train of - this time upper-branch- TSWs at point G, close to $\mathrm{H}_{43}$.

The two branches get very close to one another in the two-dimensional projection of figure $7(\mathrm{a})$, the closest approach corresponding to points $\mathrm{z}_{1}$ and $\mathrm{z}_{2}$ on either continuation curve. The possibility that the apparent close approach is an artifact of the low-dimensional projection cannot be discarded altogether, but the hypothesis is clearly supported by the virtually identical, to the bare eye, $\mathrm{z}_{1}$ and $\mathrm{z}_{2}$ vorticity colourmaps in figure $7(\mathrm{~b})$.

As a matter of fact, tracking the two families of MTSw solutions to slightly higher Re entails a completely different layout of the continuation branches that can only be explained through a codimension-2 bifurcation. Figure 8(a) depicts the arrangement of MTSw branches at $\mathrm{Re}=3300$. Both branches seem to have split in two at approximately $\mathrm{z}_{1}$ and $\mathrm{z}_{2}$, respectively, and each of the two resulting segments of each original branch have been glued at $\mathrm{z}_{3}$ and $\mathrm{z}_{4}$ to the homologous portion of the other branch. In this way, two new cross-branches, one joining Hopf points $\mathrm{H}_{41}$ and $\mathrm{H}_{42}$ and the other linking $\mathrm{H}_{43}$ and $\mathrm{H}_{34}$, have emerged. The labelled points on figure 8(b) are indistinguishable from the analogous points in figure fig- 


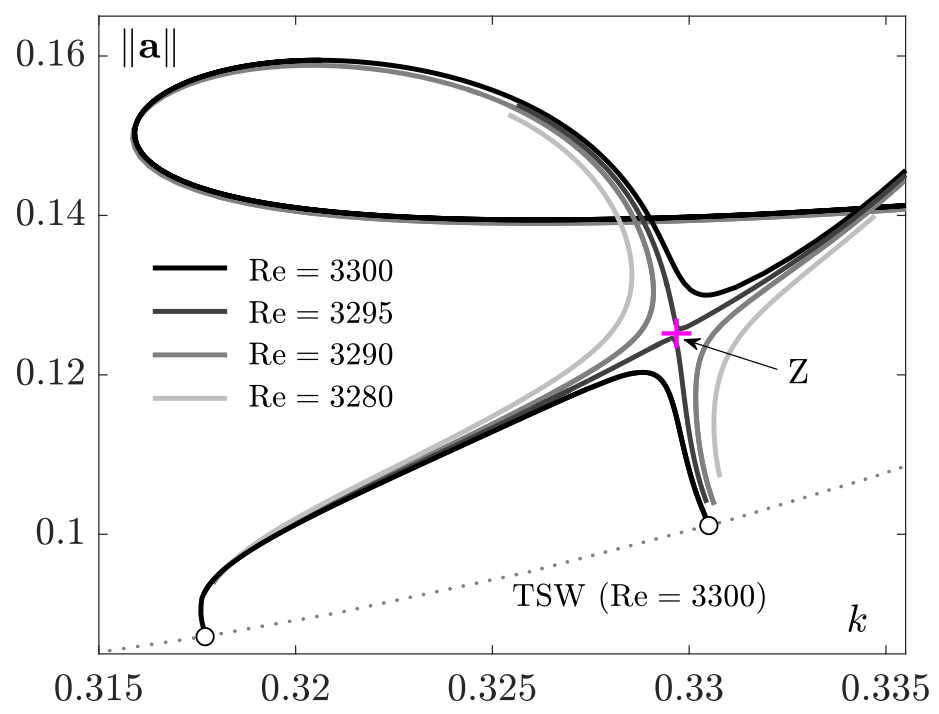

FIG. 9: Detail of codimension-2 bifurcation point $\mathrm{Z}$ (pink plus sign) relating all know types of MTSWs.

ure $7(\mathrm{~b})$ and the snapshots corresponding to $\mathrm{Z}_{i}, i=\{1,2,3,4\}$ represent perturbations of a bicritical point at $(\operatorname{Re}, k) \in([3290,3300],[0.329,0.330])$.

To better pinpoint the location of the bicritical point at which the two families of MTSWs connect, figure 9 shows a detailed continuation of solution branches within the interval $3280 \leq \operatorname{Re} \leq 3300$, in the immediate neighbourhood of the pinch point $\mathrm{Z}$. From Re $\gtrsim 3295$ on, purely upper-branch MTSWs exist in the sense that they are issued at either end from subharmonic Hopf points of upper-branch TSWs. This renders them good candidates as structural constituents of the strange saddle that governs domain-filling turbulent dynamics at sufficiently high Reynolds numbers. As such, the drifting gap that characterises them may be responsible for the wave dislocations that are typical of turbulent channel flow.

\section{CONCLUSIONS}

We have addressed in this study various unexplored aspects of symmetry breaking and streamwise modulation of propagating waves in plane Poiseuille flow. Comprehensive linear stability analysis along the upper and lower branches of TSWs has allowed identification of pitchfork bifurcations that lead to a new family of ATSW solutions that break the classical half-shift \& reflect equivariance. These new coherent states emerge from the unstable lower 
branch of TSWs at subcritical Reynolds numbers. Parametric continuation in Reynolds number Re and in streamwise wavenumber $k$ confirm that these ATsws become linearly stable within some regions of parameter space. A subharmonic Arnoldi stability analysis of the new ATSw solutions has revealed that these waves exhibit Hopf bifurcations that lead to space-modulation. The resulting time-periodic streamwise-modulated asymmetric waves (MATSW) have also been continued in parameter space, although no streamwise localisation has been identified within the ( $k, \mathrm{Re})$-range explored. They have been shown, instead, to branch off already known MTSWs bifurcated subharmonically from TSWs. While MTSWs break the TSW shift \& reflect symmetry at any given time but preserve it in the form of a space-time symmetry (they are invariant under evolution over half a period followed by a reflection about the midplane and an appropriate streamwise shift), MATSWs have completely broken all remnants of the reflection symmetry about the channel midplane. A thorough scrutiny of the potential relevance of ATSWs (and MATSWs) to the fully three-dimensional channel flow problem is far beyond the scope of the present study, yet worthwhile of a detailed exploration in future work. It is only through the disrupture of all prevailing reflectional symmetry about the channel midplane that solutions may experience a bias towards one of the walls, thereby bearing the potential for casting some light on near-wall structures observed in wall-bounded turbulent shear flows.

We have also tracked in our analysis MTSW branches emanating from lower branch TSWs through subharmonic Hopf bifurcation in the search for alternative mechanisms of streamwise localisation of travelling waves. Although no such new mechanism has been found, we have shown that these solutions are in fact related to an already known family of localising MTSWs through the occurrence of a codimension-two bifurcation point. Following this bifurcation, MTSWs exclusively interconnecting subharmonic Hopf points of upper-branch TSWs arise. These purely upper-branch solutions hold a potential for enacting wave dislocations within the strange saddle upon which domain-filling turbulent dynamics are sustained at high Reynolds numbers.

\section{Acknowledgments}

This research was supported by the Spanish MINECO grants FIS2016-77849-R, FIS201785794-P, and the Generalitat de Catalunya grant 2017-SGR-785. 


\section{Appendix A: Numerical discretization}

The Galerkin formulation of the problem consists in formally substituting expansion (9) in (7) and projecting back onto the set of Fourier-Legendre functions (10) by means of the hermitian inner product between two arbitrary fields $F(x, y)$ and $G(x, y)$ defined within the domain $\Omega=[0, \Lambda] \times[-1,1]$ by

$$
(F, G)_{\Omega} \doteq \int_{0}^{\Lambda} \int_{-1}^{1} F^{*} G \mathrm{~d} y \mathrm{~d} x .
$$

This inner product is numerically approximated using spectrally accurate de-aliased Fast Fourier Transform in the streamwise direction $x$, along with Gauss-Legendre quadrature formulas in the wall-normal coordinate $y$. The aforementioned projection is therefore

$$
\left(\Psi_{p q}, \partial_{t} \nabla^{2} \widetilde{\Psi}_{L M}\right)_{\Omega}=\left(\Psi_{p q}, \mathbf{L}_{\mathrm{Re}}\left(\widetilde{\Psi}_{L M}\right)+\mathbf{N}\left(\widetilde{\Psi}_{L M}\right)\right)_{\Omega}
$$

for $0 \leq p \leq L$ and $0 \leq q \leq M$, which leads to a system of nonlinear ordinary differential equations for the Fourier-Legendre coefficients of the form

$$
\mathbb{A}_{p q}^{\ell m} \dot{a}_{\ell m}=\mathbb{B}_{p q}^{\ell m} a_{\ell m}+\mathbb{N}(\mathbf{a})
$$

where we have used the convention of summation with respect to repeated indices, the dot in $\dot{a}_{\ell m}$ denotes a time derivative, and $\mathbb{A}_{p q}^{\ell m}, \mathbb{B}_{p q}^{\ell m}$ and $\mathbb{N}_{p q}$ are the inner products

$$
\begin{aligned}
& \mathbb{A}_{p q}^{\ell m}=\left(\Psi_{p q}, \nabla^{2} \Psi_{\ell m}\right)_{\Omega}, \\
& \mathbb{B}_{p q}^{\ell m}=\left(\Psi_{p q}, \mathbf{L}_{\operatorname{Re}}\left(\Psi_{\ell m}\right)\right)_{\Omega}, \\
& \mathbb{N}_{p q}=\left(\Psi_{p q}, \mathbf{N}\left(\widetilde{\Psi}_{L M}\right)\right)_{\Omega},
\end{aligned}
$$

for $0 \leq p, l \leq L$ and $0 \leq q, m \leq M$. The inner product in (A6) is evaluated pseudospectrally using a de-aliased grid in the streamwise direction. For simplicity, we write (A3) as

$$
\mathbb{A} \dot{\mathbf{a}}=\mathbb{B} \mathbf{a}+\mathbb{N}(\mathbf{a}) .
$$

Equation $(\mathrm{A} 7)$ constitutes an $(L+1) \times(M+1)$-dimensional nonlinear dynamical system for the Fourier-Legendre amplitudes of the disturbance. The time integration is carried out using a $4^{\text {th }}$-order IMEX method (BDF4 backward-differences linearly-implicit method for 
the linear biharmonic term and $4^{\text {th }}$-order explicit extrapolation of the nonlinear term) with associated linear multistep formula

$$
\begin{aligned}
&(25 \mathbb{A}-12 \Delta t \mathbb{B}) \mathbf{a}^{(j+1)}= \\
& \mathbb{A}\left(48 \mathbf{a}^{(j)}-36 \mathbf{a}^{(j-1)}+16 \mathbf{a}^{(j-2)}-3 \mathbf{a}^{(j-3)}\right) \\
& \quad+\Delta t\left(48 \mathbb{N}^{(j)}-72 \mathbb{N}^{(j-1)}+48 \mathbb{N}^{(j-2)}-12 \mathbb{N}^{(j-3)}\right),
\end{aligned}
$$

duly initialized by means of a $4^{\text {th }}$-order Runge-Kutta method.

A time step $\Delta t=10^{-2}$ has been found to provide sufficient accuracy for the most numerically demanding integrations herein and therefore used throughout all parametric explorations. For the spatial discretization, a minimum of $M=50$ Legendre polynomials along with $L=19 \times n$ Fourier modes ( $n$ is the number of Tollmien-Schlichting waves that can be fitted in the periodic domain) in the wall-normal and streamwise directions have been used, respectively. Neither increasing the wall-normal or streamwise spatial resolutions nor decreasing the time-step has resulted in any quantitatively significant impact on the dynamical properties of any of the flow regimes studied in this work.

\section{Appendix B: Computation and stability analysis of travelling waves}

After introducing (15) in (9), the Galerkin projection (A2) leads to a time-independent nonlinear algebraic system of equations for the unknown speed $c$ and complex state vector $\mathbf{a}^{\mathrm{TW}}=\left\{a_{\ell m}^{\mathrm{TW}}\right\}$

$$
\left(\mathbb{B}_{p q}^{\ell m}+\mathrm{i} k_{0} \ell c \mathbb{A}_{p q}^{\ell m}\right) a_{\ell m}^{\mathrm{TW}}+\mathbb{N}_{p q}\left(\mathbf{a}^{\mathrm{TW}}\right)=0
$$

for $0 \leq p \leq L$ and $0 \leq q \leq M$. System (B1) must be completed with an extra condition on the phase of the solution to remove streamwise degeneracy, leading to an algebraic system of nonlinear equations that we simply write as

$$
\mathbf{F}\left(\mathbf{a}^{\mathrm{TW}}, c ; \operatorname{Re}, k_{0}\right)=\mathbf{0}
$$

The previous system implicitly determines the sought-after coefficient vector $\mathbf{a}^{\mathrm{TW}}$ of the wave and its corresponding travelling speed $c$ as functions of the two parameters, namely the Reynolds number Re and the streamwise periodic length $\Lambda=2 \pi / k_{0}$ of the computational domain as dictated by the fundamental wave number $k_{0}$, that is

$$
\mathbf{a}^{\mathrm{TW}}=\mathbf{a}^{\mathrm{TW}}\left(\operatorname{Re}, k_{0}\right), \quad c=c\left(\operatorname{Re}, k_{0}\right) .
$$


We solve (B2) by means of Jacobian-free Newton-Krylov methods [41] and track solution branches through $\left(\mathrm{Re}, k_{0}\right)$ parameter space using pseudoarclength continuation schemes [42].

A spatially-periodic travelling wave may become unstable through many different mechanisms. If the wave preserves some symmetry of the PPF problem, a steady pitchfork bifurcation in the moving reference frame of the wave may lead to symmetry-conjugate travellingwave solution branches that break the symmetry. Alternatively, a Hopf bifurcation, be it symmetry-breaking or symmetry-preserving, may introduce a time-periodic modulation of the wave amplitude. Spatially-periodic waves may become unstable to perturbations of their same wavelength following a so-called superharmonic bifurcation, but perturbations of wavelengths longer than that of the original wave must also be considered in general, as instabilities can also be spatially-modulational as a result of a subharmonic instability.

In the most general case, the linear stability analysis of a travelling-wave solution of wavelength $\lambda_{0}=2 \pi / k_{0}$ must consider infinitesimal perturbations of wavelength $\lambda=2 \pi / k$, where in principle $k$ can take any positive real value in the interval $\left[0, k_{0}\right]$. For $k / k_{0}=m / n$, $n \in \mathbb{N}$ and $m=1, \ldots, n$, the instability of a travelling wave of wavenumber $k_{0}$ can be understood as the superharmonic instability of a train of $n$ replicas of the travelling wave $\left(\mathrm{R}_{n}\right)$, filling a periodic domain of length $\Lambda=n \lambda_{0}$, to periodic perturbations that fit $m$ times in the domain $[43,44]$. Floquet theory analysis shows that modes with $m=j$ and $m=n-j$ are related by conjugation, so that only half the possible wavelengths need to be explored. Furthermore, an analysis for a given $k / k_{0}$ can be performed on the minimal domain $n k_{0}$, such that $n / m$ expresses an irreducible fraction. Instability to irrational $k / k_{0}$ can only be interpreted in an infinitely long domain.

The representation of $a_{\ell^{\prime} m}^{\mathrm{TW}}$ in the $n$-fold replicated domain $\Omega_{n}=[-1,1] \times\left[0, n \lambda_{0}\right], n \in \mathbb{N}$ is given by

$$
a_{\ell m}(t)=a_{\ell m}^{\mathrm{TW}_{n}} \mathrm{e}^{-\mathrm{i} \ell k_{n} c t}
$$

where $k_{n}=k_{0} / n$ and $a_{\ell m}^{\mathrm{TW}}=a_{\ell^{\prime} m}^{\mathrm{TW}}$ for $\ell=n \ell^{\prime}$ and 0 otherwise. Linear stability is then analysed by considering infinitesimal disturbances to the travelling wave:

$$
a_{\ell m}(t)=\left(a_{\ell m}^{\mathrm{Tw}}+\varepsilon_{\ell m} \mathrm{e}^{\sigma t}\right) \mathrm{e}^{-\mathrm{i} \ell k_{n} c t} .
$$

Substituting (B5) in (A3) and neglecting $O\left(\varepsilon_{\ell m}^{2}\right)$ terms leads to a generalized eigenvalue 
problem of the form

$$
\left(\sigma-\mathrm{i} k_{n} \ell c\right) \mathbb{A}_{p q}^{\ell m} \varepsilon_{\ell m}=\mathbb{B}_{p q}^{\ell m} \varepsilon_{\ell m}+D_{\mathbf{a}} \mathbb{N}_{p q}^{\ell m}\left(\mathbf{a}^{\mathrm{Tw}}\right) \varepsilon_{\ell m}
$$

where $D_{\mathbf{a}} \mathbb{N}_{p q}^{\ell m}\left(\mathbf{a}^{\mathrm{TW}_{n}}\right)$ is the linearization of $\mathbb{N}$ in a neighbourhood of $\mathbf{a}^{\mathrm{TW}_{n}}$ and $(p, q)=[0, n L] \times$ $[0, M]$. For small or moderate values of $n$, the spectrum of eigenvalues $\sigma^{(j)}$ and associated

eigenvectors $\varepsilon^{(j)}=\left\{\varepsilon_{\ell m}^{(j)}\right\}$ can be solved using direct eigenvalue methods such as QZ. However, for large values of $n$, producing the full spectrum becomes unfeasible. As a matter of fact, the computation of the whole spectrum is generally unnecessary, as only the largest real part (leading) eigenvalues need to be monitored in order to identify potential bifurcations. Then, Arnoldi iteration combined with suitable Cayley transformations in the complex plane can be employed instead [45]. A comprehensive subharmonic linear stability analysis to all possible perturbation wavelengths would still incur unaffordable computational time-scales. Following MM15, we have limited the exploration to a range of wave numbers $k$ that can be analysed in domains of length up to $11 \lambda_{0}$. This streamwise periodic domain is sufficiently long to initiate a streamwise modulation that effectively localises when continued to longer domains.

\section{Appendix C: Computation of time-periodic travelling waves}

In order to accurately compute time-periodic travelling-wave solutions (PTw) to any desired accuracy, we look for fixed points of the Poincaré map $\mathbb{P}$

$$
\mathbf{a} \mapsto \tilde{\mathbf{a}}=\mathbb{P}(\mathbf{a})=\mathbb{T}(\Delta x) \Phi^{T}(\mathbf{a}),
$$

where $\Phi^{t}$ is the flow generated by (A7) and

$$
[\mathbb{T}(\Delta x) \mathbf{a}]_{\ell m}=\mathrm{e}^{-\mathrm{i} \ell k \Delta x} a_{\ell m}
$$

is the streamwise-shift operator that deals with the degeneracy in the $x$-coordinate. The time-period $T$ of the orbit and the streamwise drift $\Delta x$ must satisfy

$$
\begin{aligned}
{[\mathbb{P}(\mathbf{a})-\mathbf{a}] \cdot \widehat{\mathbb{T}}(\mathbf{a}) } & =0 \\
{[\mathbb{P}(\mathbf{a})-\mathbf{a}] \cdot \dot{\mathbf{a}} } & =0
\end{aligned}
$$

where

$$
[\widehat{\mathbb{T}}(\mathbf{a})]_{\ell m}=-\mathrm{i} \ell k a_{\ell m}
$$


is the group orbit tangent and

$$
\dot{\mathbf{a}}=\mathbb{A}^{-1}[\mathbb{B} \mathbf{a}+\mathbb{N}(\mathbf{a})]
$$

is the rate of change of the state vector. The orthogonality conditions (C3) simultaneously define the Poincaré section and remove the drift degeneracy along the group orbit. Once this degeneracy has been removed, a time-periodic travelling wave $\mathbf{a}^{\text {PTw }}$ is a fixed point of the Poincaré map $(\mathrm{C} 1)$ or, equivalently, a solution to

$$
\mathbb{P}\left(\mathbf{a}^{\mathrm{PTW}}, T ; \operatorname{Re}, k_{0}\right)-\mathbf{a}^{\mathrm{PTW}}=0,
$$

where the map $\mathbb{P}$ depends on Re and $k_{0}$ through the operator $\mathbb{B}$ involved in $\Phi^{t}$ through the time integration of (A7). Nonlinear system (C6) implicitly determines the sought PTW and its period as implicit functions of Re and $k_{0}$, that is

$$
\mathbf{a}^{\mathrm{PTW}}=\mathbf{a}^{\mathrm{PTw}}\left(\operatorname{Re}, k_{0}\right), \quad T=T\left(\operatorname{Re}, k_{0}\right) .
$$

We solve system (C6) by means of a Newton-Krylov-Poincaré method already successfully applied by MM15. Branches of PTWs can then be tracked in the $\left(\mathrm{Re}, k_{0}\right)$ parameter space using pseudoarclength continuation schemes.

[1] F. Mellibovsky and A. Meseguer, A mechanism for streamwise localisation of nonlinear waves in shear flows. J. Fluid Mech. 779, R1 (2015).

[2] P. J. Schmid and D. Henningson, Stability and transition in shear flows. Springer (2001).

[3] L. S. Tuckerman, M. Chantry, and D. Barkley, Patterns in Wall-Bounded Shear Flows. Ann. Rev. Fluid Mech. 52, 343 (2020).

[4] V. Romanov, Stability of plane-parallel Couette flow. Funct. Anal. Its Appl. 7, 137 (1973).

[5] A. Meseguer and L. N. Trefethen, Linearized pipe flow to Reynolds number 107. J. Comput. Phys. 186, 178 (2003).

[6] B. Hof, C. W. H. van Doorne, J. Westerweel, N. F. T. M., H. Holger Faisst, B. Eckhardt, H. Wedin, R. R. Kerswell, and F. Waleffe, Experimental Observation of Nonlinear Traveling Waves in Turbulent Pipe Flow. Science 305, 1594 (2004).

[7] H. Faisst and B. Eckhardt, Transition from the Couette-Taylor system to the plane Couette system. Phys. Rev. E 61(6), 7227 (2000). 
[8] H. Wedin and R. R. Kerswell, Exact coherent structures in pipe flow: travelling wave solutions. J. Fluid Mech. 508, 333 (2004).

[9] F. Mellibovsky and A. Meseguer, Critical threshold in pipe flow transition. Phil. Trans. Roy. Soc. Lond. A 367, 449 (2009).

[10] M. Nagata, Three-dimensional finite-amplitude solutions in plane Couette flow: Bifurcation from infinity. J. Fluid Mech. 217, 519 (1990).

[11] D. Viswanath, Recurrent motions within plane Couette turbulence. J. Fluid Mech. 580, 339 (2007).

[12] T. Schneider, J. Gibson, and J. Burke, Snakes and ladders: Localized solutions of plane Couette flow. Phys. Rev. Lett. 104 (2010).

[13] T. Kreilos and B. Eckhardt, Periodic orbits near onset of chaos in plane Couette flow. Chaos 22, 047505 (2012).

[14] J. F. Gibson and T. M. Schneider, Homoclinic snaking in plane Couette flow: Bending, skewing and finite-size effects. J. Fluid Mech. 794, 530 (2016).

[15] S. Zammert and B. Eckhardt, Streamwise and doubly-localised periodic orbits in plane Poiseuille flow. J. Fluid Mech. 761, 348 (2014).

[16] K. Avila, D. Moxey, A. de Lozar, M. Avila, D. Barkley, and B. Hof, The Onset of Turbulence in Pipe Flow. Science 333, 192 (2011).

[17] D. Borrero-Echeverry, M. F. Schatz, and R. Tagg, Transient turbulence in Taylor-Couette flow. Phys. Rev. E 81, 25301 (2010).

[18] P. Ritter, F. Mellibovsky, and M. Avila, Emergence of spatio-temporal dynamics from exact coherent solutions in pipe flow. New Journal of Physics 18, 083031 (2016).

[19] D. Barkley and L. S. Tuckerman, Computational Study of Turbulent Laminar Patterns in Couette Flow. Phys. Rev. Lett. 94, 014502 (2005).

[20] A. Meseguer, F. Mellibovsky, M. Avila, and F. Marques, Instability mechanisms and transition scenarios of spiral turbulence in Taylor-Couette flow. Phys. Rev. E 80, 046315 (2009).

[21] D. Samanta, A. de Lozar, and B. Hof, Experimental investigation of laminar turbulent intermittency in pipe flow. J. Fluid Mech. 681, 193 (2011).

[22] F. Dauchot, O.; Daviaud, Finite amplitude perturbation and spots growth mechanism in plane Couette flow. Phys. Fluids 7, 335 (1995).

[23] F. Mellibovsky, A. Meseguer, T. M. Schneider, and B. Eckhardt, Transition in Localized Pipe 
Flow Turbulence. Phys. Rev. Lett. 103, 054502 (2009).

[24] M. Avila, F. Mellibovsky, N. Roland, and B. Hof, Streamwise-Localized Solutions at the Onset of Turbulence in Pipe Flow. Phys. Rev. Lett. 110, 224502 (2013).

[25] M. Chantry, A. Willis, and R. R. Kerswell, Genesis of Streamwise-Localized Solutions from Globally Periodic Traveling Waves in Pipe Flow. Phys. Rev. Lett. 112 (2014).

[26] J. Barnett, D. R. Gurevich, and R. O. Grigoriev, Streamwise localization of traveling wave solutions in channel flow. Phys. Rev. E 95, 033124 (2017).

[27] J. Pugh and P. Saffman, Two-dimensional superharmonic stability of finite-amplitude waves in plane Poiseuille flow. J. Fluid Mech. 194, 295 (1988).

[28] B. Hof, A. Juel, and T. Mullin, Scaling of the turbulence transition threshold in a pipe. Phys. Rev. Lett. 91(24), 244502 (2003).

[29] L. H. Thomas, The stability of plane poiseuille flow. Phys. Rev. (Series I) 91, 780 (1953).

[30] S. A. Orszag, Numerical simulation of incompressible flows within simple boundaries: Accuracy. J. Fluid Mech. 49, 75 (1971).

[31] T. S. Chen and D. D. Joseph, Subcritical bifurcation of plane Poiseuille flow. J. Fluid Mech. 58, 337 (1973).

[32] J. P. Zahn, J. Toomre, E. Spiegel, and D. Gough, Nonlinear cellular motions in Poiseuille channel flow. J. Fluid Mech. 64, 319 (1974).

[33] U. Ehrenstein and W. Koch, Three-dimensional wavelike equilibrium states in plane Poiseuille flow. J. Fluid Mech. 228, 111 (1991).

[34] P. Casas and A. Jorba, Hopf bifurcations to quasi-periodic solutions for the two-dimensional plane Poiseuille flow. Comm. Nonlinear Sci. Num. Simul. 17, 2864 (2012).

[35] I. Soibelman and D. I. Meiron, Finite-amplitude bifurcations in plane Poiseuille flow: Twodimensional Hopf bifurcation. J. Fluid Mech. 229, 389 (1991).

[36] A. Drissi, M. Net, and I. Mercader, Subharmonic instabilities of Tollmien-Schlichting waves in two-dimensional Poiseuille flow. Phys. Rev. E 60, 1781 (1999).

[37] J. Burke and E. Knobloch, Localized states in the generalized Swift-Hohenberg equation. Phys. Rev. E 73, 056211 (2006).

[38] J. Burke and E. Knobloch, Snakes and ladders: Localized states in the Swift-Hohenberg equation. Phys. Lett. A 360, 681 (2007).

[39] M. Chantry and R. R. Kerswell, Localization in a spanwise-extended model of plane Couette 
flow. Phys. Rev. E 91, 043005 (2015).

[40] T. Price and Y. Brachet, M. Pomeau, Numerical characterization of localized solutions in plane Poiseuille flow. Phys. Fluids A 5, 762 (1993).

[41] C. Kelley, Solving nonlinear equations with Newton's method. SIAM (2003).

[42] Y. A. Kuznetsov, Elements of Applied Bifurcation Theory. Springer, third edition (2004).

[43] J. Prat, I. Mercader, and E. Knobloch, Resonant mode interactions in Rayleigh-Bénard convection. Phys. Rev. E 58, 3145 (1998).

[44] K. Melnikov, K. T., and B. Eckhardt, Long-wavelength instability of coherent structures in plane Couette flow. Phys. Rev. E 89, 043008 (2014).

[45] L. N. Trefethen and D. Bau, Numerical Linear Algebra. SIAM, first edition (1997). 